\title{
Nonparametric Bootstrap Procedures for Predictive Inference Based on Recursive Estimation Schemes*
}

\author{
Valentina Corradi ${ }^{1}$ and Norman R. Swanson ${ }^{2}$ \\ ${ }^{1}$ Queen Mary, University of London and ${ }^{2}$ Rutgers University
}

August 2005

\begin{abstract}
Our objectives in this paper are twofold. First, we introduce block bootstrap techniques that are (first order) valid in recursive estimation frameworks. Thereafter, we present two examples where predictive accuracy tests are made operational using our new bootstrap procedures. In one application, we outline a consistent test for out-of-sample nonlinear Granger causality, and in the other we outline a test for selecting amongst multiple alternative forecasting models, all of which are possibly misspecified. More specifically, our examples extend the White (2000) reality check to the case of non vanishing parameter estimation error, and extend the integrated conditional moment tests of Bierens $(1982,1990)$ and Bierens and Ploberger (1997) to the case of out-of-sample prediction. In both examples, appropriate re-centering of the bootstrap score is required in order to ensure that the tests have asymptotically correct size, and the need for such re-centering is shown to arise quite naturally when testing hypotheses of predictive accuracy. In a Monte Carlo investigation, we compare the finite sample properties of our block bootstrap procedures with the parametric bootstrap due to Kilian (1999); all within the context of various encompassing and predictive accuracy tests. An empirical illustration is also discussed, in which it is found that unemployment appears to have nonlinear marginal predictive content for inflation.
\end{abstract}

JEL classification: C22, C51.

Keywords: block bootstrap, recursive estimation scheme, reality check, nonlinear causality, parameter estimation error.

* Valentina Corradi, Department of Economics, Queen Mary, University of London, Mile End Road, London E1 4NS, UK, v.corradi@qmul.ac.uk. Norman R. Swanson, Department of Economics, Rutgers University, 75 Hamilton Street, New Brunswick, NJ 08901, USA, nswanson@econ.rutgers.edu. We owe a great many thanks to the editor, Frank Schorfheide, and two referees for numerous useful suggestions, all of which we feel were instrumental in improving earlier versions of this paper. Additionally, we wish to thank Jean-Marie Dufour, Silvia Goncalves, Stephen Gordon, Clive Granger, Oliver Linton, Brendan McCabe, Antonio Mele, Andrew Patton, Rodney Strachan, Christian Schluter, Allan Timmerman, and seminar participants at Cornell University, the London School of Economics, Laval University, Queen Mary, University of London, CIREQ-Universite' de Montreal, the University of Liverpool, Southampton University, the University of Virginia, the 2004 Winter Meetings of the Econometric Society, and the Bank of Canada for useful comments and suggestions. Corradi gratefully acknowledges financial support via ESRC grant RES-000-23-0006, and Swanson thanks the Rutgers University Research Council for financial support. 


\section{Introduction}

Economic models are often compared in terms of their relative predictive accuracy. Thus, it is not surprising that a large literature on the topic has developed over the last 10 years, including, for example, important papers by Diebold and Mariano (DM: 1995), West (1996), and White (2000). In this literature, it is quite common to compare multiple models (which are possibly all misspecified, in the sense that they are all approximations of some unknown true model) in terms of their in- or out-of-sample predictive accuracy, for a given loss function. In such contexts, one often compares parametric models containing estimated parameters. Hence, it is important to take into account the contribution of parameter estimation error when carrying out inference. Though some authors make a case in favor of using in-sample predictive evaluation (important contributions in this area include Inoue and Kilian $(2004,2005))$, it is common practice to split samples of size $T$ into $T=R+P$ observations, where only the last $P$ observations are used for predictive evaluation, particularly if all models are assumed to be (possibly) misspecified. We consider such a setup, and assume that parameters are estimated in recursive fashion, such that $R$ observations are used to construct a first parameter estimator, say $\widehat{\theta}_{R}$, a first prediction (say a 1-step ahead prediction), and a first prediction error. Then, $R+1$ observations are used to construct $\widehat{\theta}_{R+1}$, yielding a second ex ante prediction and prediction error. This procedure is continued until a final estimator is constructed using $T-1$ observations, resulting in a sequence of $P=T-R$ estimators, predictions, and prediction errors. If $R$ and $P$ grow at the same rate as the sample size, the limiting distributions of predictive accuracy tests using this setup generally reflects the contribution of parameter uncertainty (i.e. the contribution of $\frac{1}{\sqrt{P}} \sum_{t=R}^{T-1}\left(\widehat{\theta}_{t}-\theta^{\dagger}\right)$, where $\widehat{\theta}_{t}$ is a recursive $m$-estimator constructed using the first $t$ observations, and $\theta^{\dagger}$ is its probability limit, say). ${ }^{1}$ Given this fact, our objectives in this paper are twofold. First, we introduce block bootstrap techniques that are (first order) valid in recursive estimation frameworks. Thereafter, we present two examples where predictive accuracy tests are made operational using our new bootstrap procedures. One of the examples involves constructing a consistent test for out-of-sample nonlinear Granger causality, and the other involves constructing a test for selecting amongst multiple alternative forecasting models, all of which may be misspecified.

In some circumstances, such as when constructing Diebold and Mariano (1995) tests for equal

\footnotetext{
${ }^{1} m$-estimators include least squares, nonlinear least square, (quasi) maximum likelihood, and exactly identified instrumental variables and generalized method of moments estimators.
} 
(pointwise) predictive accuracy of two models, the limiting distribution is a normal random variable. In such cases, accounting for the contribution of parameter estimation error can be often be done using the framework of West (1996), and essentially involves estimating an "extra" covariance term. However, in other circumstances, such as when constructing tests which have power against generic alternatives, test statistics have limiting distributions that can be shown to be functionals of Gaussian processes with covariance kernels that reflect both (dynamic) misspecification as well as the contribution of (recursive) parameter estimation error. Such limiting distributions are not nuisance parameter free, and critical values cannot be tabulated. However, valid asymptotic critical values can be obtained via appropriate application of the (block) bootstrap. This requires a bootstrap procedure that allows for the formulation of statistics which properly mimic the contribution of $\frac{1}{\sqrt{P}} \sum_{t=R}^{T-1}\left(\widehat{\theta}_{t}-\theta^{\dagger}\right)$ (i.e. parameter estimation error). The first objective of this paper is thus to suggest a block bootstrap procedure which is valid for recursive $m$-estimators, in the sense that its use suffices to mimic the limiting distribution of $\frac{1}{\sqrt{P}} \sum_{t=R}^{T-1}\left(\widehat{\theta}_{t}-\theta^{\dagger}\right)$.

When forming the block bootstrap for recursive $m$-estimators, it is important to note that earlier observations are used more frequently than temporally subsequent observations when forming test statistics. On the other hand, in the standard block bootstrap, all blocks from the original sample have the same probability of being selected, regardless of the dates of the observations in the blocks. Thus, the bootstrap estimator, say $\widehat{\theta}_{t}^{*}$, which is constructed as a direct analog of $\widehat{\theta}_{t}$, is characterized by a location bias that can be either positive or negative, depending on the sample that we observe. In order to circumvent this problem, we suggest a re-centering of the bootstrap score which ensures that the new bootstrap estimator, which is no longer the direct analog of $\widehat{\theta}_{t}$, is asymptotically unbiased. It should be noted that the idea of re-centering is not new in the bootstrap literature for the case of full sample estimation. In fact, re-centering is necessary, even for first order validity, in the case of overidentified generalized method of moments (GMM) estimators (see e.g. Hall and Horowitz (1996), Andrews (2002, 2004), and Inoue and Shintani (2004)). This is due to the fact that, in the overidentified case, the bootstrap moment conditions are not equal to zero, even if the population moment conditions are. However, in the context of $m$-estimators using the full sample, re-centering is needed only for higher order asymptotics, but not for first order validity, in the sense that the bias term is of smaller order than $T^{-1 / 2}$ (see e.g. Andrews (2002)). In the case of recursive $m$-estimators, on the other hand, the bias term is instead of order $T^{-1 / 2}$, so that it does contribute to the limiting distribution. This points to a need for re-centering when using 
recursive estimation schemes, and such re-centering is discussed in the next section.

The block bootstrap for recursive $m$-estimators that we develop can be used to provide valid critical values in a variety of interesting testing contexts, two such leading examples of which are developed in this paper. As mentioned above, the first is a generalization of the reality check test of White (2000) that allows for non vanishing parameter estimation error. The second is an out-of-sample version of the integrated conditional moment (ICM) test of Bierens $(1982,1990)$ and Bierens and Ploberger (1997), which yields out-of-sample tests that are consistent against generic (nonlinear) alternatives. ${ }^{2}$ More specifically, our first application concerns the reality check of White (2000), which itself extends the Diebold and Mariano (1995) and West (1996) test for the relative predictive accuracy of two models by allowing for the joint comparison of multiple misspecified models against a given benchmark. The idea of White (2000) is to compare all competing models simultaneously, thus taking into account any correlation across the various models. In this context, the null hypothesis is that no competing model can outperform the benchmark, for a given loss function. As this test is usually carried out by comparing predictions from various alternative models, and given that predictions are usually formed using recursively estimated models, the issue of parameter estimation uncertainty arises naturally. White (2000) obtains valid asymptotic critical values for his test via use of the Politis and Romano (1994) stationary bootstrap for the case in which parameter estimation error is asymptotically negligible. This is the case in which either the same loss function is used for both estimation and model evaluation, or $P$ grows at a slower rate than $R$. Using the block bootstrap for recursive $m$-estimators, we generalize the reality check to the case in which parameter estimation error does not vanish asymptotically.

The objective of the second application is to test the predictive accuracy of a given (non)linear model against generic (non)linear alternatives. In particular, one chooses a benchmark model, and the objective is to test whether there is an alternative model which can provide more accurate, loss function specific, out-of-sample predictions. The test is based on a continuum of moment conditions and is consistent against generic alternatives, as discussed in Corradi and Swanson (CS: 2002). The CS test differs from the ICM test developed by Bierens (1982,1990) and Bierens and Ploberger (1997) because parameters are estimated recursively, out-of-sample prediction models are analyzed, and the null hypothesis is that the reference model delivers the best "loss function specific"

\footnotetext{
${ }^{2} \mathrm{An}$ application to predictive density and confidence interval forecast evaluation is given in Corradi and Swanson (2005).
} 
predictor, for a given information set. Given that the test compares out-of-sample prediction models, it can be viewed as a test for (non)linear out-of-sample Granger causality. Although the CS test examined in this application is the same as that discussed in Corradi and Swanson (2002), it should be noted that they use a conditional $p$-value method for constructing critical values, extending earlier work by Hansen (1996) and Inoue (2001). However, the conditional $p$ value approach suffers from the fact that under the alternative, the simulated statistics diverges at rate $\sqrt{\tilde{l}}$, conditional on the sample, where $\widetilde{l}$ plays a role analogous to the block length in the block bootstrap. This feature clearly leads to reduced power in finite samples, as shown in Corradi and Swanson (2002). As an alternative to the conditional $p$-value approach, we establish in our second example that the bootstrap for recursive $m$-estimators yields $\sqrt{P}$-consistent CS tests.

In order to shed evidence on the usefulness of the recursive block bootstrap, we carry out a Monte Carlo investigation that compares the finite sample properties of our block bootstrap procedures with two alternative naive block bootstraps as well as the parametric bootstrap due to Kilian (1999); all within the context of the CS test and a simpler non-generic version of the CS test due to Chao, Corradi and Swanson (CCS: 2001). In addition, various other related tests, including the standard F-test as well as tests due to Diebold and Mariano (1995) and Clark and McCracken (2004) are included in our Monte Carlo experiments. Results suggest that our recursive block bootstrap outperforms alternative naive nonparametric block bootstraps. Additionally, the Kilian bootstrap is shown to be robust to nonlinear misspecification, and all of the test statistics examined are found to have good finite sample properties when applied in situations where there is model misspecification.

An empirical illustration is also discussed, in which it is found that unemployment appears to have nonlinear marginal predictive content for inflation, as evidenced by application of the CS and CCS test. Findings based upon the other tests mentioned above are also presented, and it turns out that much can be learned by using all of the 5 tests in consort with one another. Namely, the different tests have different power properties against different alternatives, and hence the use of several of them can yield insight into the nature of the misspecification driving test rejection. In particular, the picture that emerges when only a subset of the tests is used to analyze the marginal predictive content of unemployment for inflation is that of an absence of predictive ability. When all of the tests are used, on the other hand, interesting evidence arises concerning the potential nonlinear predictive content of unemployment. Thus, the tests discussed in this illustration appear 
to be useful complements to each other.

The rest of the paper is organized as follows. Section 2 outlines the block bootstrap for recursive $m$-estimators and contains asymptotic results. Sections 3 and 4 contain the two examples mentioned above. Monte Carlo findings are discussed in Section 5, and our empirical illustration is presented in Section 6. Finally, concluding remarks are given in Section 7. All proofs are collected in an appendix. Hereafter, $P^{*}$ denotes the probability law governing the resampled series, conditional on the sample, $E^{*}$ and $V a r^{*}$ are the mean and variance operators associated with $P^{*}, o_{P}^{*}(1)$ $\operatorname{Pr}-P$ denotes a term converging to zero in $P^{*}$-probability, conditional on the sample, and for all samples except a subset with probability measure approaching zero, and $O_{P}^{*}(1) \operatorname{Pr}-P$ denotes a term which is bounded in $P^{*}$-probability, conditional on the sample, and for all samples except a subset with probability measure approaching zero. Analogously, $O_{a . s .}{ }^{(1)}$ and $o_{a . s . *}(1)$ denote terms that are almost surely bounded and terms that approach zero almost surely, according the the probability law $P^{*}$, and conditional on the sample. Note that $P$ is also used to denote the length of the prediction period, and unless otherwise obvious from the context in which it is used, clarification of the meaning is given.

\section{The Block Bootstrap for Recursive $m$-Estimators}

In this section, we establish the first order validity of a block bootstrap estimator that captures the effect of parameter estimation error in the context of recursive $m$-estimators, which are defined as follows. Let $Z^{t}=\left(y_{t}, \ldots, y_{t-s_{1}+1}, X_{t}, \ldots, X_{t-s_{2}+1}\right), t=1, \ldots, T$, and let $s=\max \left\{s_{1}, s_{2}\right\}$. Additionally, assume that $i=1, \ldots, n$ models are estimated (thus allowing us to establish notation that will be useful in the applications presented in subsequent sections). Now, define the recur sive $m$-estimator for the parameter vector associated with model $i$ as: ${ }^{3}$

$$
\widehat{\theta}_{i, t}=\arg \min _{\theta_{i} \in \Theta_{i}} \frac{1}{t} \sum_{j=s}^{t} q_{i}\left(y_{j}, Z^{j-1}, \theta_{i}\right), \quad R \leq t \leq T-1, i=1, \ldots, n
$$

and

$$
\theta_{i}^{\dagger}=\arg \min _{\theta_{i} \in \Theta_{i}} E\left(q_{i}\left(y_{j}, Z^{j-1}, \theta_{i}\right)\right)
$$

\footnotetext{
${ }^{3}$ Within the context of full sample estimation, the first order validity of the block bootstrap for $m$-estimators has been shown by Goncalves and White (2004), for dependent and heterogeneous series.
} 
where $q_{i}$ denotes the objective function for model $i$. As the discussion below does not depend on any specific model, we drop the subscript $i$. Following standard practice (such as in the real-time forecasting literature), this estimator is first computed using $R$ observations. In our applications we focus on 1-step ahead prediction (although results can be extended quite easily to multiple step ahead prediction), and so that recursive estimators are thus subsequently computed using $R+1$ observations, and then $R+2$ observations, and so on, until the last estimator is constructed using $T-1$ observations. This results in a sequence of $P=T-R$ estimators. These estimators can then used to construct sequences of $P$ 1-step ahead forecasts and associated forecast errors, for example.

Now, consider the overlapping block resampling scheme of Künsch (1989), which can be applied in our context as follows: ${ }^{4}$ At each replication, draw $b$ blocks (with replacement) of length $l$ from the sample $W_{t}=\left(y_{t}, Z^{t-1}\right)$, where $b l=T-s$. Thus, the first block is equal to $W_{i+1}, \ldots, W_{i+l}$, for some $i=s-1, \ldots, T-l+1$, with probability $1 /(T-s-l+1)$, the second block is equal to $W_{i+1}, \ldots, W_{i+l}$, again for some $i=s-1, \ldots, T-l+1$, with probability $1 /(T-s-l+1)$, and so on, for all blocks, where the block length grows with the sample size at an appropriate rate. More formally, let $I_{k}, k=1, \ldots, b$ be $i i d$ discrete uniform random variables on $[s-1, s, \ldots, T-l+1]$. Then, the resampled series, $W_{t}^{*}=$ $\left(y_{t}^{*}, Z^{*, t-1}\right)$, is such that $W_{1}^{*}, W_{2}^{*}, \ldots, W_{l}^{*}, W_{l+1}^{*}, \ldots, W_{T}^{*}=W_{I_{1}+1}, W_{I_{1}+2}, \ldots, W_{I_{1}+l}, W_{I_{2}+1}, \ldots, W_{I_{b}+l}$, and so a resampled series consists of $b$ blocks that are discrete iid uniform random variables, conditional on the sample.

Suppose we define the bootstrap estimator, $\widehat{\theta}_{t}^{*}$, to be the direct analog of $\widehat{\theta}_{t}$. Namely,

$$
\widehat{\theta}_{t}^{*}=\arg \min _{\theta \in \Theta} \frac{1}{t} \sum_{j=s}^{t} q\left(y_{j}^{*}, Z^{*, j-1}, \theta\right), \quad R \leq t \leq T-1 .
$$

By first order conditions, $\frac{1}{t} \sum_{j=s}^{t} \nabla_{\theta} q\left(y_{j}^{*}, Z^{*, j-1}, \widehat{\theta}_{t}^{*}\right)=0$, and via a mean value expansion of

\footnotetext{
${ }^{4}$ The main difference between the block bootstrap and the stationary bootstrap of Politis and Romano (PR:1994) is that the former uses a deterministic block length, which may be either overlapping as in Künsch (1989) or nonoverlapping as in Carlstein (1986), while the latter resamples using blocks of random length. One important feature of the PR bootstrap is that the resampled series, conditional on the sample, is stationary, while a series resampled from the (overlapping or non overlapping) block bootstrap is nonstationary, even if the original sample is strictly stationary. However, Lahiri (1999) shows that all block boostrap methods, regardless of whether the block length is deterministic or random, have a first order bias of the same magnitude, but the bootstrap with deterministic block length has a smaller first order variance. In addition, the overlapping block boostrap is more efficient than the non overlapping block bootstrap.
} 
$\frac{1}{t} \sum_{j=s}^{t} \nabla_{\theta} q\left(y_{j}^{*}, Z^{*, j-1}, \widehat{\theta}_{t}^{*}\right)$ around $\widehat{\theta}_{t}$, after a few simple manipulations, we have that

$$
\begin{aligned}
& \frac{1}{\sqrt{P}} \sum_{t=R}^{T-1}\left(\widehat{\theta}_{t}^{*}-\widehat{\theta}_{t}\right) \\
= & \frac{1}{\sqrt{P}} \sum_{t=R}^{T-1}\left(\left(-\frac{1}{t} \sum_{j=s}^{t} \nabla_{\theta}^{2} q\left(y_{j}^{*}, Z^{*, j-1}, \bar{\theta}_{t}^{*}\right)\right)^{-1} \frac{1}{t} \sum_{j=s}^{t} \nabla_{\theta} q\left(y_{j}^{*}, Z^{*, j-1}, \widehat{\theta}_{t}\right)\right) \\
= & B^{\dagger} \frac{1}{\sqrt{P}} \sum_{t=R}^{T-1}\left(\frac{1}{t} \sum_{j=s}^{t} \nabla_{\theta} q\left(y_{j}^{*}, Z^{*, j-1}, \widehat{\theta}_{t}\right)\right)+o_{P^{*}}(1) \operatorname{Pr}-P \\
= & B^{\dagger} \frac{a_{R, 0}}{\sqrt{P}} \sum_{j=s}^{R} \nabla_{\theta} q\left(y_{j}^{*}, Z^{*, j-1}, \widehat{\theta}_{R}\right)+B^{\dagger} \frac{1}{\sqrt{P}} \sum_{j=1}^{P-1} a_{R, j} \nabla_{\theta} q\left(y_{R+j}^{*}, Z^{*, R+j-1}, \widehat{\theta}_{R+j}\right) \\
& +o_{P^{*}}(1) \operatorname{Pr}-P,
\end{aligned}
$$

where $\bar{\theta}_{t}^{*} \in\left(\widehat{\theta}_{t}^{*}, \widehat{\theta}_{t}\right), B^{\dagger}=E\left(-\nabla_{\theta}^{2} q\left(y_{j}, Z^{j-1}, \theta^{\dagger}\right)\right)^{-1}, a_{R, j}=\frac{1}{R+j}+\frac{1}{R+j+1}+\ldots+\frac{1}{R+P-1}, j=$ $0,1, \ldots, P-1$, and where the last equality on the right hand side of (4) follows immediately, using the same arguments as those used in Lemma A5 of West (1996). Analogously,

$$
\begin{aligned}
& \frac{1}{\sqrt{P}} \sum_{t=R}^{T-1}\left(\widehat{\theta}_{t}-\theta^{\dagger}\right) \\
= & B^{\dagger} \frac{a_{R, 0}}{\sqrt{P}} \sum_{j=s}^{R} \nabla_{\theta} q\left(y_{j}, Z^{j-1}, \theta^{\dagger}\right)+B^{\dagger} \frac{1}{\sqrt{P}} \sum_{j=1}^{P-1} a_{R, j} \nabla_{\theta} q\left(y_{R+j}, Z^{R+j-1}, \theta^{\dagger}\right)+o_{P}(1) .
\end{aligned}
$$

Now, given (2), $E\left(\nabla_{\theta} q\left(y_{j}, Z^{j-1}, \theta^{\dagger}\right)\right)=0$ for all $j$, and $\frac{1}{\sqrt{P}} \sum_{t=R}^{T-1}\left(\widehat{\theta}_{t}-\theta^{\dagger}\right)$ has a zero mean normal limiting distribution (see Theorem 4.1 in West (1996)). On the other hand, as any block of observations has the same chance of being drawn,

$$
E^{*}\left(\nabla_{\theta} q\left(y_{j}^{*}, Z^{*, j-1}, \widehat{\theta}_{t}\right)\right)=\frac{1}{T-s} \sum_{k=s}^{T-1} \nabla_{\theta} q\left(y_{k}, Z^{k-1}, \widehat{\theta}_{t}\right)+O\left(\frac{l}{T}\right) \operatorname{Pr}-P,
$$

where the $O\left(\frac{l}{T}\right)$ term arises because the first and last $l$ observations have a lesser chance of being drawn (see e.g. Fitzenberger (1997)). ${ }^{5}$ Now, $\frac{1}{T-s} \sum_{k=s}^{T-1} \nabla_{\theta} q\left(y_{k}, Z^{k-1}, \widehat{\theta}_{t}\right) \neq 0$, and is instead of order $O_{P}\left(T^{-1 / 2}\right)$. Thus, $\frac{1}{\sqrt{P}} \sum_{t=R}^{T-1} \frac{1}{T-s} \sum_{k=s}^{T-1} \nabla_{\theta} q\left(y_{k}, Z^{k-1}, \widehat{\theta}_{t}\right)=O_{P}(1)$, and does not vanish in probability. This clearly contrasts with the full sample case, in which $\frac{1}{T-s} \sum_{k=s}^{T-1} \nabla_{\theta} q\left(y_{k}, Z^{k-1}, \widehat{\theta}_{T}\right)=$ 0 , because of the first order conditions. Thus, $\frac{1}{\sqrt{P}} \sum_{t=R}^{T-1}\left(\widehat{\theta}_{t}^{*}-\widehat{\theta}_{t}\right)$ cannot have a zero mean normal

\footnotetext{
${ }^{5}$ In fact, the first and last observation in the sample can appear only at the beginning and end of the block, for example.
} 
limiting distribution, but is instead characterized by a location bias that can be either positive or negative depending on the sample.

Given (6), our objective is thus to have the bootstrap score centered around $\frac{1}{T-s} \sum_{k=s}^{T-1} \nabla_{\theta} q\left(y_{k}, Z^{k-1}, \widehat{\theta}_{t}\right)$. Hence, define a new bootstrap estimator, $\widetilde{\theta}_{t}^{*}$, as:

$$
\widetilde{\theta}_{t}^{*}=\arg \min _{\theta \in \Theta} \frac{1}{t} \sum_{j=s}^{t}\left(q\left(y_{j}^{*}, Z^{*, j-1}, \theta\right)-\theta^{\prime}\left(\frac{1}{T} \sum_{k=s}^{T-1} \nabla_{\theta} q\left(y_{k}, Z^{k-1}, \widehat{\theta}_{t}\right)\right)\right)
$$

$R \leq t \leq T-1 .^{6}$

Now, note that first order conditions are $\frac{1}{t} \sum_{j=s}^{t}\left(\nabla_{\theta} q\left(y_{j}^{*}, Z^{*, j-1}, \widetilde{\theta}_{t}^{*}\right)-\left(\frac{1}{T} \sum_{k=s}^{T-1} \nabla_{\theta} q\left(y_{k}, Z^{k-1}, \widehat{\theta}_{t}\right)\right)\right)=$ 0 ; and via a mean value expansion of $\frac{1}{t} \sum_{j=s}^{t} \nabla_{\theta} q\left(y_{j}^{*}, Z^{*, j-1}, \widetilde{\theta}_{t}^{*}\right)$ around $\widehat{\theta}_{t}$, after a few simple manipulations, we have that:

$$
\begin{aligned}
& \frac{1}{\sqrt{P}} \sum_{t=R}^{T-1}\left(\widetilde{\theta}_{t}^{*}-\widehat{\theta}_{t}\right) \\
= & B^{\dagger} \frac{1}{\sqrt{P}} \sum_{t=R}^{T}\left(\frac{1}{t} \sum_{j=s}^{t}\left(\nabla_{\theta} q\left(y_{j}^{*}, Z^{*, j-1}, \widehat{\theta}_{t}\right)-\left(\frac{1}{T} \sum_{k=s}^{T-1} \nabla_{\theta} q\left(y_{k}, Z^{k-1}, \widehat{\theta}_{t}\right)\right)\right)\right) \\
& +o_{P^{*}}(1), \operatorname{Pr}-P .
\end{aligned}
$$

Thus, given (6), it is immediate to see that the bias associated with $\frac{1}{\sqrt{P}} \sum_{t=R}^{T-1}\left(\widetilde{\theta}_{t}^{*}-\widehat{\theta}_{t}\right)$ is of order $O\left(l T^{-1 / 2}\right)$, conditional on the sample, and so it is negligible for first order asymptotics, as $l=o\left(T^{1 / 2}\right)$.

Theorem 1, which summarizes these results, requires the following assumptions.

Assumption A1: $\left(y_{t}, X_{t}\right)$, with $y_{t}$ scalar and $X_{t}$ an $\Re^{\zeta}$-valued $(0<\zeta<\infty)$ vector, is a strictly stationary and absolutely regular $\beta$-mixing process with size $-4(4+\psi) / \psi, \psi>0$.

Assumption A2: (i) $\theta^{\dagger}$ is uniquely identified (i.e. $E\left(q\left(y_{t}, Z^{t-1}, \theta\right)\right)>E\left(q\left(y_{t}, Z^{t-1}, \theta^{\dagger}\right)\right)$ for any $\theta \neq \theta^{\dagger}$ ); (ii) $q$ is twice continuously differentiable on the interior of $\Theta$, and for $\Theta$ a compact subset of $\Re^{\varrho}$; (iii) the elements of $\nabla_{\theta} q$ and $\nabla_{\theta}^{2} q$ are $p$-dominated on $\Theta$, with $p>2(2+\psi)$, where $\psi$ is the same positive constant as defined in Assumption A1; and (iv) $E\left(-\nabla_{\theta}^{2} q(\theta)\right)$ is negative definite uniformly on $\Theta .^{7}$

\footnotetext{
${ }^{6}$ More precisely, we should use $\frac{1}{t-s}$ and $\frac{1}{T-s}$ to scale the summand in (7). For notational simplicity, $\frac{1}{t-s}$ and $\frac{1}{T-s}$ are approximated with $\frac{1}{t}$ and $\frac{1}{T}$.

${ }^{7}$ We say that $\nabla_{\theta} q\left(y_{t}, Z^{t-1}, \theta\right)$ is $2 r$-dominated on $\Theta$ if its $j-t h$ element, $j=1, \ldots, \varrho$, is such that $\left|\nabla_{\theta} q\left(y_{t}, Z^{t-1}, \theta\right)\right|_{j} \leq D_{t}$, and $E\left(\left|D_{t}\right|^{2 r}\right)<\infty$. For more details on domination conditions, see Gallant and White (1988, pp. 33).
} 
Assumption A3: $T=R+P$, and as $T \rightarrow \infty, P / R \rightarrow \pi$, with $0<\pi<\infty$.

Assumptions A1 and A2 are standard memory, moment, smoothness and identifiability conditions. A1 requires $\left(y_{t}, X_{t}\right)$ to be strictly stationary and absolutely regular. The memory condition is stronger than $\alpha$-mixing, but weaker than (uniform) $\phi$-mixing. Assumption A3 requires that $R$ and $P$ grow at the same rate. In fact, if $P$ grows at a slower rate than $R$, i.e. $P / R \rightarrow 0$, then

$\frac{1}{\sqrt{P}} \sum_{t=R}^{T}\left(\widehat{\theta}_{t}-\theta^{\dagger}\right)=o_{P}(1)$ and so there were no need to capture the contribution of parameter estimation error.

Theorem 1: Let A1-A3 hold. Also, assume that as $T \rightarrow \infty, l \rightarrow \infty$, and that $\frac{l}{T^{1 / 4}} \rightarrow 0$. Then, as $T, P$ and $R \rightarrow \infty$,

$$
P\left(\omega: \sup _{v \in \Re \varrho}\left|P_{T}^{*}\left(\frac{1}{\sqrt{P}} \sum_{t=R}^{T}\left(\widetilde{\theta}_{t}^{*}-\widehat{\theta}_{t}\right) \leq v\right)-P\left(\frac{1}{\sqrt{P}} \sum_{t=R}^{T}\left(\widehat{\theta}_{t}-\theta^{\dagger}\right) \leq v\right)\right|>\varepsilon\right) \rightarrow 0,
$$

where $P_{T}^{*}$ denotes the probability law of the resampled series, conditional on the (entire) sample.

Broadly speaking, Theorem 1 states that $\frac{1}{\sqrt{P}} \sum_{t=R}^{T-1}\left(\widetilde{\theta}_{t}^{*}-\widehat{\theta}_{t}\right)$ has the same limiting distribution as $\frac{1}{\sqrt{P}} \sum_{t=R}^{T-1}\left(\widehat{\theta}_{t}-\theta^{\dagger}\right)$, conditional on sample, and for all samples except a set with probability measure approaching zero. As outlined in the following sections, application of Theorem 1 allows us to capture the contribution of (recursive) parameter estimation error to the covariance kernel of the limiting distribution of various statistics. If Assumption 3 is violated and $P / R \rightarrow 0$, then the statement in the theorem above is trivially satisfied, in the sense that both $\frac{1}{\sqrt{P}} \sum_{t=R}^{T}\left(\widetilde{\theta}_{t}^{*}-\widehat{\theta}_{t}\right)$ and $\frac{1}{\sqrt{P}} \sum_{t=R}^{T}\left(\widehat{\theta}_{t}-\theta^{\dagger}\right)$ have a limiting distribution degenerate on zero.

\section{The Reality Check}

In this section, we extend the White (2000) reality check to the case in which the effect of parameter estimation error does not vanish asymptotically. In particular, we show that the block bootstrap for recursive $m$-estimators properly mimics the contribution of parameter estimation error to the covariance kernel of the limiting distribution of the original reality check test. Although we focus our attention in this paper on the block bootstrap, which is based on resampling blocks of deterministic length, we conjecture that the same approach can be used to extend the stationary bootstrap employed by White (2000) to the case of nonvanishing parameter estimation error.

Let the forecast error be $u_{i, t+1}=y_{t+1}-\kappa_{i}\left(Z^{t}, \theta_{i}^{\dagger}\right)$, and let $\widehat{u}_{i, t+1}=y_{t+1}-\kappa_{i}\left(Z^{t}, \widehat{\theta}_{i, t}\right)$, where $\kappa_{i}\left(Z^{t}, \widehat{\theta}_{i, t}\right)$ is the estimated conditional mean function under model $i$. Also, assume that the set 
of regressors may vary across different models, so that $Z^{t}$ is meant to denote the collection of all potential regressors. Following White (2000), define the statistic $S_{P}=\max _{i=2, \ldots, n} S_{P}(1, i)$, where

$$
S_{P}(1, i)=\frac{1}{\sqrt{P}} \sum_{t=R}^{T-1}\left(g\left(\widehat{u}_{1, t+1}\right)-g\left(\widehat{u}_{i, t+1}\right)\right), i=2, \ldots, n,
$$

and where $g$ is a given loss function (see Christoffersen and Diebold $(1996,1997)$ and Elliott and Timmerman $(2004 \mathrm{a}, \mathrm{b})$ for a detailed discussion of loss functions used in predictive evaluation) ${ }^{8}$ Recall that in this test, parameter estimation error need not be accounted for in the covariance kernel of the limiting distribution unless $g \neq q_{i}$ for some $i$. This follows upon examination of the results of both West (1996) and White (2000). In particular, in West (1996), the parameter estimation error components that enter into the covariance kernel of the limiting distribution of his test statistic are zero whenever the same loss function is used for both predictive evaluation and in-sample estimation. The same argument holds for the reality check test. This means that as long as $g=q_{i} \forall i$, the White test can be applied regardless of the rate of growth of $P$ and $R$. When we write the covariance kernel of the limiting distribution of the statistic (see below), however, we include terms capturing the contribution of parameter estimation error, thus implicitly assuming that $g \neq q_{i}$ for some $i$. In practice, one reason why we allow for cases where $g \neq q_{i}$ is that least squares is sometimes better behaved in finite samples and/or easier to implement than more generic $m$-estimators, so that practitioners sometimes use least squares for estimation and more complicated (possibly asymmetric) loss functions for predictive evaluation. ${ }^{9}$ Of course, there are also applications for which parameter estimation error does not vanish, even if the same loss function is used for parameter estimation and predictive evaluation. One such application is discussed in the next section.

For a given loss function, the reality check tests the null hypothesis that a benchmark model (defined as model 1) performs equal to or better than all competitor models (i.e. models $2, \ldots, n$ ). The alternative is that at least one competitor performs better than the benchmark. ${ }^{10}$ Formally,

\footnotetext{
${ }^{8}$ See Hansen (2004) for further discussion of the White (2004) test.

${ }^{9}$ Consider linex loss, where $g(u)=e^{a u}-a u-1$, so that for $a>0(a<0)$ positive (negative) errors are more (less) costly than negative (positive) errors. Here, the errors are exponentiated, so that in this particular case, laws of large numbers and central limit theorems may require a large number of observations before providing satisfactory approximations. This feature of linex loss is illustrated in the Monte Carlo findings of Corradi and Swanson (2002). (Linex loss is studied in Zellner (1986), Christoffersen and Diebold (1996, 1997) and Granger (1999), for example.)

${ }^{10}$ In the current context, we are interested in choosing the model which is more accurate for given loss function. An
} 
the hypotheses are:

$$
H_{0}: \max _{i=2, \ldots, n} E\left(g\left(u_{1, t+1}\right)-g\left(u_{i, t+1}\right)\right) \leq 0
$$

and

$$
H_{A}: \max _{i=2, \ldots, n} E\left(g\left(u_{1, t+1}\right)-g\left(u_{i, t+1}\right)\right)>0 .
$$

In order to derive the limiting distribution of $S_{P}$ we require the following additional assumption.

Assumption A4: (i) $\kappa_{i}$ is twice continuously differentiable on the interior of $\Theta_{i}$ and the elements of $\nabla_{\theta_{i}} \kappa_{i}\left(Z^{t}, \theta_{i}\right)$ and $\nabla_{\theta_{i}}^{2} \kappa_{i}\left(Z^{t}, \theta_{i}\right)$ are $p$-dominated on $\Theta_{i}$, for $i=2, \ldots, n$, with $p>2(2+\psi)$, where $\psi$ is the same positive constant as that defined in Assumption A1; (ii) $g$ is positive valued, twice continuously differentiable on $\Theta_{i}$, and $g, g^{\prime}$ and $g^{\prime \prime}$ are $p$-dominated on $\Theta_{i}$ with $p$ defined as in (i); and (iii) let $S_{g_{i} g_{i}}=\lim _{T \rightarrow \infty} \operatorname{Var}\left(\frac{1}{\sqrt{T}} \sum_{t=s}^{T}\left(g\left(u_{1, t+1}\right)-g\left(u_{i, t+1}\right)\right)\right), i=2, \ldots, n$, define analogous covariance terms, $S_{g_{j} g_{k}}, j, k=2, \ldots, n$, and assume that $\left[S_{g_{j} g_{k}}\right]$ is positive semi-definite.

Assumptions A4(i)-(ii) are standard smoothness and domination conditions imposed on the conditional mean functions of the models. Assumption A4(iii) is standard in the literature that uses DM type tests (see e.g. West (1996) and White (2000)), and states that at least one of the competing models has to be nonnested with (and not nesting) the benchmark.

Proposition 1: Let Assumptions A1-A4 hold. Then, as $P, R \rightarrow \infty$,

$$
\max _{i=2, \ldots, n}\left(S_{P}(1, i)-\sqrt{P} E\left(g\left(u_{1, t+1}\right)-g\left(u_{i, t+1}\right)\right)\right) \stackrel{d}{\rightarrow} \max _{i=2, \ldots, n} S(1, i),
$$

where $S=(S(1,2), \ldots, S(1, n))$ is a zero mean Gaussian process with covariance kernel given by $V$, with $V$ a $n \times n$ matrix with $i, i$ element

$v_{i, i}=S_{g_{i} g_{i}}+2 \Pi \mu_{1}^{\prime} B_{1}^{\dagger} C_{11} B_{1}^{\dagger} \mu_{1}+2 \Pi \mu_{i}^{\prime} B_{i}^{\dagger} C_{i i} B_{i}^{\dagger} \mu_{i}-4 \Pi \mu_{1}^{\prime} B_{1}^{\dagger} C_{1 i} B_{i}^{\dagger} \mu_{i}+2 \Pi S_{g_{i q_{1}}} B_{1}^{\dagger} \mu_{1}-2 \Pi S_{g_{i q_{i}}} B_{i}^{\dagger} \mu_{i}$, where $S_{g_{i} g_{i}}$ is defined as in Assumption A4(iii), $C_{i i}=\sum_{\tau=-\infty}^{\infty} E\left(\left(\nabla_{\theta_{i}} q_{i}\left(y_{1+s}, Z^{s}, \theta_{i}^{\dagger}\right)\right)\left(\nabla_{\theta i} q_{i}\left(y_{1+s+\tau}, Z^{s+\tau}, \theta_{i}^{\dagger}\right)\right)^{\prime}\right)$, $S_{g_{i q_{i}}}=\sum_{\tau=-\infty}^{\infty} E\left(\left(g\left(u_{1,1}\right)-g\left(u_{i, 1}\right)\right)\left(\nabla_{\theta i} q_{i}\left(y_{1+s+\tau}, Z^{s+\tau}, \theta_{i}^{\dagger}\right)\right)^{\prime}\right)$, $B_{i}^{\dagger}=\left(E\left(-\nabla_{\theta i}^{2} q_{i}\left(y_{t}, Z^{t-1}, \theta_{i}^{\dagger}\right)\right)\right)^{-1}, \mu_{i}=E\left(\nabla_{\theta_{i}} g\left(u_{i, t+1}\right)\right)$, and $\Pi=1-\pi^{-1} \ln (1+\pi)$. Just as in White (2000), note that under the null, the least favorable case arises when $E\left(g\left(u_{1, t+1}\right)-g\left(u_{i, t+1}\right)\right)=0, \forall i$. In this case, the distribution of $S_{P}$ coincides with that of $\max _{i=2, \ldots, n}\left(S_{P}(1, i)-\sqrt{P} E\left(g\left(u_{1, t+1}\right)-g\left(u_{i, t+1}\right)\right)\right)$, so that $S_{P}$ has the above limiting distribalternative approach is to combine different forecasting models in some optimal way. For very recent contributions along these lines, see Elliott and Timmermann (2004a,b). 
ution, which is a functional of a Gaussian process with a covariance kernel that reflects uncertainty due to parameter estimation error and dynamic misspecification. Additionally, when all competitor models are worse than the benchmark, the statistic diverges to minus infinity at rate $\sqrt{P}$. Finally, when only some competitor models are worse than the benchmark, the limiting distribution provides a conservative test, as $S_{P}$ will always be smaller than

$\max _{i=2, \ldots, n}\left(S_{P}(1, i)-\sqrt{P} E\left(g\left(u_{1, t+1}\right)-g\left(u_{i, t+1}\right)\right)\right)$, asymptotically. Of course, when $H_{A}$ holds, the statistic diverges to plus infinity at rate $\sqrt{P}$.

Recall that the maximum of a Gaussian process is not Gaussian in general, so that standard critical values cannot be used to conduct inference on $S_{P}$. Our approach to this problem is to apply the block bootstrap for recursive $m$-estimators outlined above. ${ }^{11}$

Define the bootstrap parameter estimator as:

$$
\widetilde{\theta}_{i, t}^{*}=\arg \min _{\theta_{i} \in \Theta_{i}} \frac{1}{t} \sum_{j=s}^{t}\left(q_{i}\left(y_{j}^{*}, Z^{*, j-1}, \theta_{i}\right)-\theta_{i}^{\prime}\left(\frac{1}{T} \sum_{h=s}^{T-1} \nabla_{\theta_{i}} q_{i}\left(y_{h}, Z^{h-1}, \widehat{\theta}_{i, t}\right)\right)\right),
$$

where $R \leq t \leq T-1, i=1, \ldots, n$. Further, define the bootstrap statistic as $S_{P}^{*}=\max _{i=2, \ldots, n} S_{P}^{*}(1, i)$, where:

$$
\begin{aligned}
S_{P}^{*}(1, i)= & \frac{1}{\sqrt{P}} \sum_{t=R}^{T-1}\left[\left(g\left(y_{t+1}^{*}-\kappa_{1}\left(Z^{*, t}, \widetilde{\theta}_{1, t}^{*}\right)\right)-g\left(y_{t+1}^{*}-\kappa_{i}\left(Z^{*, t}, \widetilde{\theta}_{i, t}^{*}\right)\right)\right)\right. \\
& \left.-\left\{\frac{1}{T} \sum_{j=s}^{T-1}\left(g\left(y_{j+1}-\kappa_{1}\left(Z^{j}, \widehat{\theta}_{1, t}\right)\right)-g\left(y_{j+1}-\kappa_{i}\left(Z^{j}, \widehat{\theta}_{i, t}\right)\right)\right)\right\}\right] .
\end{aligned}
$$

Note that bootstrap statistic in (9) is different from the "usual" bootstrap statistic, which is defined as the difference between the statistic computed over the sample observations and over the bootstrap observations. That is, following the usual approach to bootstrap statistic construction, one might have expected that the appropriate bootstrap statistic would be:

$$
\begin{aligned}
\bar{S}_{P}^{*}(1, i)= & \frac{1}{\sqrt{P}} \sum_{t=R}^{T-1}\left[\left(g\left(y_{t+1}^{*}-\kappa_{1}\left(Z^{*, t}, \widetilde{\theta}_{1, t}^{*}\right)\right)-g\left(y_{t+1}^{*}-\kappa_{i}\left(Z^{*, t}, \widetilde{\theta}_{i, t}^{*}\right)\right)\right)\right. \\
& \left.-\left(g\left(y_{t+1}-\kappa_{1}\left(Z^{t}, \widehat{\theta}_{1, t}\right)\right)-g\left(y_{t+1}-\kappa_{i}\left(Z^{t}, \widehat{\theta}_{i, t}\right)\right)\right)\right] .
\end{aligned}
$$

\footnotetext{
${ }^{11}$ For further discussion, the reader is referred to Corradi and Swanson (2004a,2005). Included in these papers, for example, is an overview of critical value construction via subsampling (see also Politis, Romano and Wolf (1999) and Linton, Maasoumi and Whang (2004)), and the use of the stationary bootstrap in cases where parameter estimation eror vanishes asymptotically (i.e. White (2000)). A Monte Carlo discussion of bootstrap methods is in Diebold and Chen (1996).
} 
Instead, as can be seen by inspection of $S_{P}^{*}(1, i)$, the bootstrap (resampled) component is constructed only over the last $P$ observations, while the sample component is constructed over all $T$ observations. Although a formal proof is provided in the appendix, it is worthwhile to give a heuristic explanation of the validity of the statistic in (9). For sake of simplicity, consider a single model, say model 1. Now,

$$
\begin{aligned}
& \frac{1}{\sqrt{P}} \sum_{t=R}^{T-1}\left(g\left(y_{t+1}^{*}-\kappa_{1}\left(Z^{*, t}, \widetilde{\theta}_{1, t}^{*}\right)\right)-\frac{1}{T} \sum_{j=s}^{T-1} g\left(y_{j+1}-\kappa_{1}\left(Z^{j}, \widehat{\theta}_{1, t}\right)\right)\right) \\
= & \frac{1}{\sqrt{P}} \sum_{t=R}^{T-1}\left(g\left(y_{t+1}^{*}-\kappa_{1}\left(Z^{*, t}, \widehat{\theta}_{1, t}\right)\right)-\frac{1}{T} \sum_{j=s}^{T-1} g\left(y_{j+1}-\kappa_{1}\left(Z^{j}, \widehat{\theta}_{1, t}\right)\right)\right) \\
+ & \frac{1}{\sqrt{P}} \sum_{t=R}^{T-1} \nabla_{\theta} g\left(y_{t+1}^{*}-\kappa_{1}\left(Z^{*, t}, \bar{\theta}_{1, t}^{*}\right)\right)\left(\widetilde{\theta}_{1, t}^{*}-\widehat{\theta}_{1, t}\right),
\end{aligned}
$$

where $\bar{\theta}_{1, t}^{*} \in\left(\widetilde{\theta}_{1, t}^{*}, \widehat{\theta}_{1, t}\right)$. Notice that the first term on the RHS of (11) mimics the limiting behavior of $\frac{1}{\sqrt{P}} \sum_{t=R}^{T-1}\left(g\left(u_{1, t+1}\right)-E\left(g\left(u_{1, t+1}\right)\right)\right)$, while the second term mimics the limiting behavior of the parameter estimation error associated with model 1 . Needless to say, the same holds for any arbitrary model. This leads to the following theorem.

Theorem 2: Let Assumptions A1-A4 hold. Also, assume that as $T \rightarrow \infty, l \rightarrow \infty$, and that $\frac{l}{T^{1 / 4}} \rightarrow 0$. Then, as $T, P$ and $R \rightarrow \infty$,

$$
P\left(\omega: \sup _{v \in \Re}\left|P_{T}^{*}\left(\max _{i=2, \ldots, n} S_{P}^{*}(1, i) \leq v\right)-P\left(\max _{i=2, \ldots n} S_{P}^{\mu}(1, i) \leq v\right)\right|>\varepsilon\right) \rightarrow 0,
$$

and

$$
S_{P}^{\mu}(1, i)=S_{P}(1, i)-\sqrt{P} E\left(g\left(u_{1, t+1}\right)-g\left(u_{i, t+1}\right)\right),
$$

The above result suggests proceeding in the following manner. For any bootstrap replication, compute the bootstrap statistic, $S_{P}^{*}$. Perform $B$ bootstrap replications ( $B$ large) and compute the quantiles of the empirical distribution of the $B$ bootstrap statistics. Reject $H_{0}$, if $S_{P}$ is greater than the $(1-\alpha) t h$-percentile. Otherwise, do not reject. Now, for all samples except a set with probability measure approaching zero, $S_{P}$ has the same limiting distribution as the corresponding bootstrapped statistic when $E\left(g\left(u_{1, t+1}\right)-g\left(u_{i, t+1}\right)\right)=0 \forall i$, ensuring asymptotic size equal to $\alpha$. On the other hand, when one or more competitor models are strictly dominated by the benchmark, the rule provides a test with asymptotic size between 0 and $\alpha$ (see above discussion). Under the 
alternative, $S_{P}$ diverges to (plus) infinity, while the corresponding bootstrap statistic has a well defined limiting distribution, ensuring unit asymptotic power.

\section{The CS Test}

CS (2002) draw on both the consistent specification and predictive ability testing literatures in order to propose a test for predictive accuracy which is consistent against generic nonlinear alternatives, which is designed for comparing nested models, and which allows for dynamic misspecification of all models being evaluated. The CS test is an out-of-sample version of the ICM test, as discussed in the introduction of this paper. This test is relevant for model selection, as it is well known that DM and reality check tests do not have a well defined limiting distributions when the benchmark is nested with all competing models and when $P / R \rightarrow 0$ (see e.g. Corradi and Swanson (2002, 2004b), McCracken (2004) and Clark and McCracken (2004)). ${ }^{12}$ Alternative (non DM) tests for comparing the predictive ability of a fixed number of nested models have previously also been suggested. For example, Clark and McCracken (2001,2004) propose encompassing tests for comparing two nested models for one-step and multi-step ahead prediction, respectively. Giacomini and White (2003) introduce a test for conditional predictive ability that is valid for both nested and nonnested models. The key ingredient of their test is the fact that parameters are estimated using a fixed rolling window. Finally, Inoue and Rossi (2004) suggest a recursive test, where not only the parameters, but the statistic itself, are computed in a recursive manner.

The main difference between these tests and the CS test is that the CS test is consistent against generic (non)linear alternatives and not only against a fixed alternative.

Summarizing the testing approach considered in this application, assume that the objective is to test whether there exists any unknown alternative model that has better predictive accuracy than a given benchmark model, for a given loss function. A typical example is the case in which the benchmark model is a simple autoregressive model and we want to check whether a more accurate forecasting model can be constructed by including possibly unknown (non)linear functions of the

\footnotetext{
${ }^{12}$ McCracken (2004) provides a very interesting result based on a version of the DM test in which loss is quadratic and martingale difference scores are assumed (i.e. it is assumed that the model is correctly specified under the null hypothesis). In particular, he shows that in this case, the DM test has a nonstandard limiting distribution which is a functional of Brownian motions, whenever $P / R \rightarrow \pi>0$. Clark and McCracken (2004) extend McCracken (2004) to the case of multi step ahead forecasts.
} 
past of the process or of the past of some other process (e.g. out-of-sample (non)linear Granger causality tests can be constructed in this manner). ${ }^{13}$ Although this is the case that we focus on, the benchmark model can in general be any (non)linear model. One important feature of this application is that the same loss function is used for in-sample estimation and out-of-sample prediction (see Granger (1993), Weiss (1996), and Schorfheide (2004) for further discussion of this issue $)^{14}$. In contrast to the previous application, however, this does not ensure that parameter estimation error vanishes asymptotically.

Let the benchmark model be:

$$
y_{t}=\theta_{1,1}^{\dagger}+\theta_{1,2}^{\dagger} y_{t-1}+u_{1, t}
$$

where $\theta_{1}^{\dagger}=\left(\theta_{1,1}^{\dagger}, \theta_{1,2}^{\dagger}\right)^{\prime}=\arg \min _{\theta_{1} \in \Theta_{1}} E\left(q_{1}\left(y_{t}-\theta_{1,1}-\theta_{1,2} y_{t-1}\right)\right), \theta_{1}=\left(\theta_{1,1}, \theta_{1,2}\right)^{\prime}, y_{t}$ is a scalar, and $q_{1}=g$, as the same loss function is used both for in-sample estimation and out-of-sample predictive evaluation. The generic alternative model is:

$$
y_{t}=\theta_{2,1}^{\dagger}(\gamma)+\theta_{2,2}^{\dagger}(\gamma) y_{t-1}+\theta_{2,3}^{\dagger}(\gamma) w\left(Z^{t-1}, \gamma\right)+u_{2, t}(\gamma)
$$

where $\theta_{2}^{\dagger}(\gamma)=\left(\theta_{2,1}^{\dagger}(\gamma), \theta_{2,2}^{\dagger}(\gamma), \theta_{2,3}^{\dagger}(\gamma)\right)^{\prime}=\arg \min _{\theta_{2} \in \Theta_{2}} E\left(q_{1}\left(y_{t}-\theta_{2,1}-\theta_{2,2} y_{t-1}-\theta_{2,3} w\left(Z^{t-1}, \gamma\right)\right)\right)$, $\theta_{2}(\gamma)=\left(\theta_{2,1}(\gamma), \theta_{2,2}(\gamma), \theta_{2,3}(\gamma)\right)^{\prime}, \theta_{2} \in \Theta_{2}, \Gamma$ is a compact subset of $\Re^{d}$, for some finite $d$. The alternative model is called "generic" because of the presence of $w\left(Z^{t-1}, \gamma\right)$, which is a generically comprehensive function, such as Bierens' exponential, a logistic, or a cumulative distribution function (see e.g. Stinchcombe and White (1998) for a detailed explanation of generic comprehensiveness). One example has $w\left(Z^{t-1}, \gamma\right)=\exp \left(\sum_{i=1}^{s_{2}} \gamma_{i} \Phi\left(X_{t-i}\right)\right)$, where $\Phi$ is a measurable one to one mapping from $\Re$ to a bounded subset of $\Re$, so that here $Z^{t}=\left(X_{t}, \ldots, X_{t-s_{2}+1}\right)$, and we are thus testing for nonlinear Granger causality. The hypotheses of interest are:

$$
H_{0}: E\left(g\left(u_{1, t+1}\right)-g\left(u_{2, t+1}(\gamma)\right)\right)=0 \text { versus } H_{A}: E\left(g\left(u_{1, t+1}\right)-g\left(u_{2, t+1}(\gamma)\right)\right)>0
$$

Clearly, the reference model is nested within the alternative model, and given the definitions of $\theta_{1}^{\dagger}$

\footnotetext{
${ }^{13}$ For example, Swanson and White (1997) compare the predictive accuracy of various linear models against neural network models using both in-sample and out-of-sample model selection criteria.

${ }^{14}$ In the context of multi-step ahead vector autoregressive prediction, Schorfheide (2004) proposes a new prediction criterion that can be used to jointly select the number of lags as well as to choose between (quasi)-maximum likelihood estimators and loss function based estimators.
} 
and $\theta_{2}^{\dagger}(\gamma)$, the null model can never outperform the alternative. ${ }^{15}$ For this reason, $H_{0}$ corresponds to equal predictive accuracy, while $H_{A}$ corresponds to the case where the alternative model outperforms the reference model, as long as the errors above are loss function specific forecast errors. It follows that $H_{0}$ and $H_{A}$ can be restated as:

$$
H_{0}: \theta_{2,3}^{\dagger}(\gamma)=0 \text { versus } H_{A}: \theta_{2,3}^{\dagger}(\gamma) \neq 0
$$

for $\forall \gamma \in \Gamma$, except for a subset with zero Lebesgue measure. Now, given the definition of $\theta_{2}^{\dagger}(\gamma)$, note that

$$
E\left(g^{\prime}\left(y_{t+1}-\theta_{2,1}^{\dagger}(\gamma)-\theta_{2,2}^{\dagger}(\gamma) y_{t}-\theta_{2,3}^{\dagger}(\gamma) w\left(Z^{t}, \gamma\right)\right) \times\left(\begin{array}{c}
-1 \\
-y_{t} \\
-w\left(Z^{t}, \gamma\right)
\end{array}\right)\right)=0
$$

where $g^{\prime}$ is the derivative of the loss function with respect to its argument. Thus, under $H_{0}$ we have that $\theta_{2,3}^{\dagger}(\gamma)=0, \theta_{2,1}^{\dagger}(\gamma)=\theta_{1,1}^{\dagger}, \theta_{2,2}^{\dagger}(\gamma)=\theta_{1,2}^{\dagger}$, and $E\left(g^{\prime}\left(u_{1, t+1}\right) w\left(Z^{t}, \gamma\right)\right)=0$. Thus, we can once again restate $H_{0}$ and $H_{A}$ as:

$$
H_{0}: E\left(g^{\prime}\left(u_{1, t+1}\right) w\left(Z^{t}, \gamma\right)\right)=0 \text { versus } H_{A}: E\left(g^{\prime}\left(u_{1, t+1}\right) w\left(Z^{t}, \gamma\right)\right) \neq 0
$$

for $\forall \gamma \in \Gamma$, except for a subset with zero Lebesgue measure. Finally, define the forecast error as $\widehat{u}_{1, t+1}=y_{t+1}-\left(\begin{array}{ll}1 & y_{t}\end{array}\right) \widehat{\theta}_{1, t}$. Following CS, the test statistic is:

$$
M_{P}=\int_{\Gamma} m_{P}(\gamma)^{2} \phi(\gamma) d \gamma
$$

where

$$
m_{P}(\gamma)=\frac{1}{P^{1 / 2}} \sum_{t=R}^{T-1} g^{\prime}\left(\widehat{u}_{1, t+1}\right) w\left(Z^{t}, \gamma\right)
$$

and where $\int_{\Gamma} \phi(\gamma) d \gamma=1, \phi(\gamma) \geq 0$, with $\phi(\gamma)$ absolutely continuous with respect to Lebesgue measure. Note also that " 1 " denotes derivative with respect to the argument of the function. Elsewhere, we use " $\nabla_{x}$ " to denote derivative with respect to $x$. In the sequel, we require the following assumptions.

Assumption A5: (i) $w$ is a bounded, twice continuously differentiable function on the interior of $\Gamma$ and $\nabla_{\gamma} w\left(Z^{t}, \gamma\right)$ is bounded uniformly in $\Gamma$; and (ii) $\nabla_{\gamma} \nabla_{\theta_{1}} q_{1, t}^{\prime}\left(\theta_{1}\right) w\left(Z^{t-1}, \gamma\right)$ is continuous on

\footnotetext{
${ }^{15}$ Needless to say, in finite samples the forecasting mean square prediction error from the small model can be lower than that associated with the larger model. Indeed, this property arises in our empirical illustration.
} 
$\Theta_{1} \times \Gamma$, where $q_{1, t}^{\prime}\left(\theta_{1}\right)=q_{1}^{\prime}\left(y_{t}-\theta_{1,1}-\theta_{1,2} y_{t-1}\right), \Gamma$ a compact subset of $\Re^{d}$, and is $2 r$-dominated uniformly in $\Theta_{1} \times \Gamma$, with $r \geq 2(2+\psi)$, where $\psi$ is the same positive constant as that defined in Assumption A1.

Assumption A5 requires the function $w$ to be bounded and twice continuously differentiable; such a requirement is satisfied by logistic or exponential functions, for example.

Proposition 2: Let Assumptions A1-A3 and A5 hold. Then, the following results hold: (i) Under $H_{0}$

$$
M_{P}=\int_{\Gamma} m_{P}(\gamma)^{2} \phi(\gamma) d \gamma \stackrel{d}{\rightarrow} \int_{\Gamma} Z(\gamma)^{2} \phi(\gamma) d \gamma
$$

where $m_{P}(\gamma)$ is defined in equation (17) and $Z$ is a Gaussian process with covariance kernel given by:

$$
\begin{aligned}
K\left(\gamma_{1}, \gamma_{2}\right)= & S_{g g}\left(\gamma_{1}, \gamma_{2}\right)+2 \Pi \mu_{\gamma_{1}}^{\prime} B^{\dagger} S_{h h} B^{\dagger} \mu_{\gamma_{2}}+\Pi \mu_{\gamma_{1}}^{\prime} B^{\dagger} S_{g h}\left(\gamma_{2}\right) \\
& +\Pi \mu_{\gamma_{2}}^{\prime} B^{\dagger} S_{g h}\left(\gamma_{1}\right),
\end{aligned}
$$

with $\mu_{\gamma_{1}}=E\left(\nabla_{\theta_{1}}\left(g_{t+1}^{\prime}\left(u_{1, t+1}\right) w\left(Z^{t}, \gamma_{1}\right)\right)\right), B^{\dagger}=\left(E\left(\nabla_{\theta_{1}}^{2} q_{1}\left(u_{1, t}\right)\right)\right)^{-1}$, $S_{g g}\left(\gamma_{1}, \gamma_{2}\right)=\sum_{j=-\infty}^{\infty} E\left(g^{\prime}\left(u_{1, s+1}\right) w\left(Z^{s}, \gamma_{1}\right) g^{\prime}\left(u_{1, s+j+1}\right) w\left(Z^{s+j}, \gamma_{2}\right)\right)$, $S_{h h}=\sum_{j=-\infty}^{\infty} E\left(\nabla_{\theta_{1}} q_{1}\left(u_{1, s}\right) \nabla_{\theta_{1}} q_{1}\left(u_{1, s+j}\right)^{\prime}\right)$, $S_{g h}\left(\gamma_{1}\right)=\sum_{j=-\infty}^{\infty} E\left(g^{\prime}\left(u_{1, s+1}\right) w\left(Z^{s}, \gamma_{1}\right) \nabla_{\theta_{1}} q_{1}\left(u_{1, s+j}\right)^{\prime}\right)$, and $\gamma, \gamma_{1}$, and $\gamma_{2}$ are generic elements of $\Gamma$.

(ii) Under $H_{A}$, for $\varepsilon>0$, $\lim _{P \rightarrow \infty} \operatorname{Pr}\left(\frac{1}{P} \int_{\Gamma} m_{P}(\gamma)^{2} \phi(\gamma) d \gamma>\varepsilon\right)=1$.

As in the previous application, the limiting distribution under $H_{0}$ is a Gaussian process with a covariance kernel that reflects both the dependence structure of the data and the effect of parameter estimation error. Hence, critical values are data dependent and cannot be tabulated.

In order to implement this statistic using the block bootstrap for recursive $m$-estimators, define: ${ }^{16}$

$$
\begin{aligned}
\widetilde{\theta}_{1, t}^{*}= & \left(\widetilde{\theta}_{1,1, t}^{*}, \widetilde{\theta}_{1,2, t}^{*}\right)^{\prime}=\arg \min _{\theta_{1} \in \Theta_{1}} \frac{1}{t} \sum_{j=2}^{t}\left[q_{1}\left(y_{j}^{*}-\theta_{1,1}-\theta_{1,2} y_{j-1}^{*}\right)\right. \\
& \left.-\theta_{1}^{\prime} \frac{1}{T} \sum_{i=2}^{T-1} \nabla_{\theta} q_{1}\left(y_{i}-\widehat{\theta}_{1,1, t}-\widehat{\theta}_{1,2, t} y_{i-1}\right)\right]
\end{aligned}
$$

\footnotetext{
${ }^{16}$ Recall that $y_{t}^{*}, Z^{*, t}$ is obtained via the resampling procedure described in Section 2
} 
Also, define $\widetilde{u}_{1, t+1}^{*}=y_{t+1}^{*}-\left(\begin{array}{ll}1 & y_{t}^{*}\end{array}\right) \widetilde{\theta}_{1, t}^{*}$. The bootstrap test statistic is:

$$
M_{P}^{*}=\int_{\Gamma} m_{P}^{*}(\gamma)^{2} \phi(\gamma) d \gamma
$$

where, recalling that $g=q_{1}$,

$$
m_{P}^{*}(\gamma)=\frac{1}{P^{1 / 2}} \sum_{t=R}^{T-1}\left(g^{\prime}\left(y_{t+1}^{*}-\left(\begin{array}{ll}
1 & y_{t}^{*}
\end{array}\right) \widetilde{\theta}_{1, t}^{*}\right) w\left(Z^{*, t}, \gamma\right)-\frac{1}{T} \sum_{i=2}^{T-1} g^{\prime}\left(y_{i}-\left(\begin{array}{ll}
1 & y_{i-1}
\end{array}\right) \widehat{\theta}_{1, t}\right) w\left(Z^{i-1}, \gamma\right)\right)
$$

As in the reality check case, the bootstrap statistic in (19) is characterized by the fact that the bootstrap (resampled) component is constructed only over the last $P$ observations, while the sample component is constructed over all $T$ observations. The same heuristic arguments given to justify this form of bootstrap statistic in the previous application also apply here.

Theorem 3: Let Assumptions A1-A3 and A5 hold. Also, assume that as $T \rightarrow \infty, l \rightarrow \infty$, and that $\frac{l}{T^{1 / 4}} \rightarrow 0$. Then, as $T, P$ and $R \rightarrow \infty$,

$$
P\left(\omega: \sup _{v \in \Re}\left|P_{T}^{*}\left(\int_{\Gamma} m_{P}^{*}(\gamma)^{2} \phi(\gamma) d \gamma \leq v\right)-P\left(\int_{\Gamma} m_{P}^{\mu}(\gamma)^{2} \phi(\gamma) d \gamma \leq v\right)\right|>\varepsilon\right) \rightarrow 0
$$

where $m_{P}^{\mu}(\gamma)=m_{P}(\gamma)-\sqrt{P} E\left(g^{\prime}\left(u_{1, t+1}\right) w\left(Z^{t}, \gamma\right)\right)$.

The above result suggests proceeding the same way as in the first application.

\section{Monte Carlo Results}

In this section we carry out a series of Monte Carlo experiments comparing the recursive block bootstrap with a variety of other bootstraps, and comparing the finite sample performance of the test discussed above with a variety of other tests. With regard to the bootstrap, we consider 4 alternatives. Namely: (i) the "Recur Block Bootstrap", which is the block bootstrap for recursive $m$-estimators discussed above; (ii) "Block Bootstrap, no PEE, no adjust" is a strawman block bootstrap used for comparison purposes, where it is assumed that there is no parameter estimation error (PEE), so that $\widehat{\theta}_{1, t}$ is used in place of $\widetilde{\theta}_{1, t}^{*}$ in the construction of $M_{P}^{*}$, and the term $\frac{1}{T} \sum_{i=1}^{T-1} g^{\prime}\left(y_{i+1}-\left(\begin{array}{cc}1 & y_{i}\end{array}\right) \widehat{\theta}_{1, t}\right) w\left(Z^{i}, \gamma\right)$ in $m_{P}^{*}$ is replaced with $g^{\prime}\left(y_{t+1}-\left(\begin{array}{ll}1 & y_{t}\end{array}\right) \widehat{\theta}_{1, t}\right) w\left(Z^{t}, \gamma\right)$ (i.e. there is no bootstrap statistic adjustment, thus conforming with the usual case when the standard block bootstrap is used); (iii) "Standard Block Bootstrap" is the standard block bootstrap (i.e. this bootstrap is the same as that outlined in (ii), except that $\widehat{\theta}_{1, t}$ is replaced with $\widehat{\theta}_{1, t}^{*}$; and 
(iv) the parametric bootstrap of Kilian (1999), applied in the spirit of McCracken and Sapp (MS: 2004). As in MS, application of the parametric bootstrap begins with the estimation of a VAR model for $x_{t}$ and $y_{t}$ (with lags selected using the Schwarz Information Criterion (SIC)) and resample the residuals as if they were iid. Then the pseudo time series $x_{t}^{*}$ and $y_{t}^{*}$ are constructed using estimated parameters and resampled residuals, and using the original VAR structure. At this point, $x_{t}^{*}$ and $y_{t}^{*}$ are used to estimate parameters recursively and construct a series of one-step ahead prediction errors (see below). Finally, bootstrap statistics are constructed exactly as are the original statistics, except that prediction errors and variables are replaced with their bootstrapped counterparts. It should be pointed out that a necessary condition for the asymptotic validity of the Kilian (1999) parametric bootstrap is that the underlying DGP is nested by the VAR used in the bootstrap procedure. Thus, it is not in general valid if the underlying DGP contains nonlinear component(s). ${ }^{17}$ Furthermore, in our context, validity of this bootstrap still remains to be established even in the case for which the DGP is nested by the linear VAR model. This is because the bootstrap statistics are formed using one-step ahead forecast errors based on recursively estimated parameters. Therefore, standard arguments used to show the validity of the parametric bootstrap no longer apply. Nevertheless, it is interesting that the parametric bootstrap clearly still performs quite well in a variety of nonlinear contexts, as shown in the experiments reported on below.

The test statistics examined in our experiments include: (i) the standard in-sample F-test; (ii) the encompassing test due to Clark and McCracken (CM: 2004) and Harvey, Leybourne and Newbold (1997); (iii) the Diebold and Mariano (DM: 1995) test; (iv) the CS test; and (v) the CCS test. $^{18}$

\footnotetext{
${ }^{17}$ See Kilian and Taylor (2003) for a modification of this procedure in the context of specific nonlinear models. Additionally, note that even when the alternative is nonlinear, the parametric bootstrap of Kilian (1999) will have correct size asymptotically, as long as the underlying model is linear under the null. In practice, one would expect this bootstrap procedure to have power against some nonlinear alternatives, although it is not designed to have power against general nonlinear alternatives.

${ }^{18}$ The CCS statistic is essentially the same as the CS test, but uses $Z^{t}$ instead of a generically comprehensive function thereof (recall that $Z^{t}$ contains the additional variables included in the "bigger" model defined below). Thus, this test can be seen as a special case of the CS test that is designed to have power against linear alternatives, and it is not explicitely designed to have power against generic nonlinear alternatives as is the CS test. The theory in Section 4 of this paper thus applies to both the CS and CCS tests. Additionally, the CM test is included in our study because it is an encompassing test which is designed to have power against linear alternatives, and so it is directly comparable with the CCS test. Finally, the F and DM tests are included in our analysis because they are the most
} 
To be more specific, note that the CM test is an out-of-sample encompassing test, and is defined as follows:

$$
C M=(P-h+1)^{1 / 2} \frac{\frac{1}{P-h+1} \sum_{t=R}^{T-h} \widehat{c}_{t+h}}{\frac{1}{P-h+1} \sum_{j=-\bar{j}}^{\bar{j}} \sum_{t=R+j}^{T-h} K\left(\frac{j}{M}\right)\left(\widehat{c}_{t+h}-\bar{c}\right)\left(\widehat{c}_{t+h-j}-\bar{c}\right)},
$$

where $\widehat{c}_{t+h}=\widehat{u}_{1, t+h}\left(\widehat{u}_{1, t+h}-\widehat{u}_{2, t+h}\right), \bar{c}=\frac{1}{P-h+1} \sum_{t=R}^{T-\tau} \widehat{c}_{t+h}, K(\cdot)$ is a kernel (such as the Bartlett kernel), and $0 \leq K\left(\frac{j}{M}\right) \leq 1$, with $K(0)=1$, and $M=o\left(P^{1 / 2}\right)$. Additionally, $h$ is the forecast horizon (set equal to unity in our experiments), $P$ is as defined above, and $\widehat{u}_{1, t+1}$ and $\widehat{u}_{2, t+1}$ are the out-of sample forecast errors associated with least squares estimation of "smaller" and "bigger" linear models, respectively (see below for further details). Note that $\bar{j}$ does not grow with the sample size. Therefore, the denominator in $C M$ is a consistent estimator of the long run variance only when $E\left(c_{t} c_{t+|k|}\right)=0$ for all $|k|>h$ (see Assumption A3 in Clark and McCracken (2004)). Thus, the statistic takes into account the moving average structure of the multistep prediction errors, but still does not allow for dynamic misspecification under the null. This is one of the main differences between the CM and CS (CCS) tests.

Note also that the DM test is the mean square error version of the Diebold and Mariano (1995) test for predictive accuracy, and is defined as follows:

$$
D M=\sqrt{P} \frac{\frac{1}{P} \sum_{t=R}^{T} \widehat{d}_{t+h}}{\frac{1}{P-h+1} \sum_{j=-\bar{j}}^{\bar{j}} \sum_{t=R+j}^{T-h} K\left(\frac{j}{M}\right)\left(\widehat{d}_{t+h}-\bar{d}\right)\left(\widehat{d}_{t+h-j}-\bar{d}\right)},
$$

where $\widehat{d}_{t+h}=\widehat{u}_{1, t+h}^{2}-\widehat{u}_{2, t+h}^{2}$, and $\bar{d}=\frac{1}{P-h+1} \sum_{t=R}^{T-\tau} \widehat{d}_{t+h}$. The limiting distributions of the CM and DM statistics are given in Theorems 3.1 and 3.2 in Clark and McCracken (2004), and for $h>1$ contain nuisance parameters so that critical values cannot be directly tabulated, and hence Clark and McCracken (2004) use the Kilian parametric bootstrap to obtain critical values. In this case, as discussed above, it is not clear that the parametric bootstrap is asymptotically valid. However, again as alluded to above, the parametric bootstrap approach taken by Clark and McCracken is clearly a good approximation, at least for the DGPs and horizon considered in our experiments, given that these tests have very good finite sample properties (see discussion of results below).

Data are generated according to the DGPs summarized in Table 1 as : Size1-Size2 and Power1Power12.

commonly applied and examined in- and out-of-sample tests used for model selection. They thus serve as a kind of benchmark against which the performance of the other tests can be measured. 
In our setup, the benchmark model (denoted by Size1 in Table 1) is an AR(1). (The benchmark model is also called the "small" model.) The null hypothesis is that no competing model outperforms the benchmark model. Twelve of our DGPs (denoted by Power1-Power12) include (non)linear functions of $x_{t-1}$. In this sense, our focus is on (non)linear out-of-sample Granger causality testing. The parameter $a_{4}=\{0,1\}$, so that the variable $w_{t-1}$ enters into one half of the DGPs. As this regressor is never included in any regression models, it is meant to render all estimated models misspecified if $a_{4}=1$. Even in cases where $a_{4}=0$, all regression models are misspecified, as all fitted regression functions are linear in their variables (so that there is neglected nonlinear (Granger) causality). The exception to this rule is the case where data are generated according to Power2. An important further clarification of our setup is as follows. CS and CCS tests only require estimation of the benchmark models. The CM, F, and DM tests require estimation of the benchmark models as well as the alternative models. In our context, the alternative model estimated is simply the benchmark model with $x_{t-1}$ added as an additional regressor, regardless of which DGP is used to generate the data. The alternative is also sometimes called the "big" model. ${ }^{19}$

The functional forms that are specified under the alternative include: (i) exponential (Power1,Power 7$)$; (ii) linear (Power2); (iii) self exciting threshold (Power3), squared (Power8), and absolute value (Power9). In addition, Power4-Power6 and Power10-Power12 are the same as the others, except that an MA(1) term is added. Notice that Power1 includes a nonlinear term that is similar in form to the test function, $g(\cdot)$, which is defined below. Also, Power2 serves as linear causality benchmarks. Finally, Power'7 is an explosive "strawman" model. Test statistics are constructed by fitting what is referred to in the next section as a "small model" (i.e. a linear AR(1) in $y_{t}$ ) in order to construct the CS and CCS test statistics. Note that the "big model" (which is a linear AR(1) model in $y_{t}$, with $x_{t-1}$ added as an additional regressor) is only fitted in order to construct the $\mathrm{F}$, CM, and DM test statistics. It is not necessary to fit this model when constructing the CS and CCS statistics. All test statistics are formed using one-step ahead predictions (and corresponding prediction errors) from recursively estimated models.

In all experiments, we set $g\left(z^{t-1}, \gamma\right)=\exp \left(\sum_{i=1}^{2}\left(\gamma_{i} \tan ^{-1}\left(\left(z_{i, t-1}-\bar{z}_{i}\right) / 2 \widehat{\sigma}_{z_{i}}\right)\right)\right)$, with $z_{1, t-1}=$

\footnotetext{
${ }^{19}$ Note that Power 5 is also linear in $x_{t-1}$. However, corresponding fitted linear regression models with $y_{t}, y_{t-1}$, and $x_{t-1}$ are still misspecified, as there is an AR error component in the DGP that is not accounted for in the fitted models.
} 
$x_{t-1}, z_{2, t-1}=y_{t-1}$, and $\gamma_{1}, \gamma_{2}$ scalars. Additionally, define $\Gamma=[0.0,5.0] \mathrm{x}[0.0,5.0]$. We consider a grid that is delineated by increments of size 0.5. All results are based on 500 Monte Carlo replications, and samples of $T=200, T=300$, and $T=600$ are used. For the sake of brevity, however, results for $T=200$ and $T=300$ are not included and are available upon request, although there is little additional to see in these results, as power is lower for smaller sample sizes, and as all tests have empirical rejection frequencies that are fairly close to nominal test levels. ${ }^{20}$ The following parameterizations are used: $a_{1}=1.0, a_{2}=\{0.3,0.6,0.9\}$, and $a_{3}=0.3$. Additionally, bootstrap critical values are constructed using 100 simulated statistics, the block length, $l$, is set equal to $\{2,5,10\},\{4,10,20\}$, or $\{10,20,50\}$, depending upon the degree of DGP persistence, as given by the value of $a_{2}$. Finally, all results are based on $P=(1 / 2) T$ recursively formed predictions.

Findings are summarized in Tables 2-3 for the CS test, and Tables 4-5 for all other tests. Note that Tables 2 and 3 differ only with regard to the value of $a_{4}$, which is equal to 1 for the former and to 0 for the latter. The same distinction applies to Tables 4 and 5 . The first column in the tables states the DGP used to generate data (e.g. Size1). Thus, Size1-2 refer to empirical level experiments, and Power1-12 refer to empirical power experiments. All numerical entries are test rejection frequencies. Further details of the mnemonics used in the tables is contained in the footnotes to Table 2 and 4 . Although results are only reported for the case where $P=0.5 T$, additional results for $P=0.4 T$ and $0.6 T$ were also tabulated. These results are qualitatively similar to those reported, and are available upon request from the authors.

Before turning to a discussion of our findings, it should be stressed that we offer observations regarding two broad issues. First, is the recursive block bootstrap useful, or could one simply use the standard block bootstrap? To a lesser extent, we also address the issue of whether the Kilian parametric bootstrap performs favorably for the CS test discussed in Section 4 and for the other test statistics mentioned above. Second, we attempt to shed some light on the impact of model misspecification on the finite sample performance of the various tests statistics examined using our different approaches for critical value construction. To a lesser extent, we also address the issue of in-sample versus out-of-sample testing (i.e. vis a vis comparison of F-test performance with the performance of the other tests). Overall, results are quite clear-cut, and can be summarized as follows for Tables 2 and 3, where the CS test is examined.

\footnotetext{
${ }^{20}$ Of course, and as expected, block lengths must be increased as sample sizes are increased in order to retain good finite sample empirical level when using the CS and CCS tests.
} 
First, by inspection of Tables 2-3, we note immediately that, as predicted by the theoretical results stated in the previous sections, the recursive block bootstrap gives rise to tests with much better finite sample properties than the bootstrap which does not take into account parameter estimation error and the standard block bootstrap, when applied to the CS test. In particular, both incorrect nonparametric bootstrap procedures (i.e. those title "BB, no PEE, no adj" and "Block Bootstrap" - see footnote to Table 2 for further details) give rise to tests which are severely undersized, and consequently have lower power. Empirical rejection frequencies based upon the recursive block bootstrap are often close to the nominal $10 \%$ level, on the other hand. For example, in Panel A of Tables 2 and 3, empirical rejection levels for $l=10$ are 0.11 and 0.09 (Table 2) and 0.10 and 0.11 (Table 3), for Size 1 and Size 2, respectively. Recalling that models estimated in Table 3 always contain a missing variable $\left(w_{t-1}\right)$, and are thus misspecified, and recalling also that Size 2 experiments involve estimating models with unmodelled error serial correlation, it appears also that the recursive block bootstrap handles (missing variable) misspecification quite well. However, it is clear that in the presence high levels of dependence in the lagged endogenous variable in our models (e.g. $a_{2}=0.6$ or 0.9 ), one should use large block lengths, as expected. ${ }^{21}$ Furthermore, even when larger block lengths are used in such cases, the empirical levels reported (see Panels B and C of Tables 2 and 3) are quite low relative to the nominal level, with values often ranging from around 0.03-0.08. Of course, use of the incorrect nonparametric bootstraps leads to rejection frequencies of 0.00 in many of these cases. Notice also in Panels B and C of Tables 2 and 3 that CS tests constructed using data generated according to Size 2 yields poorer empirical level performance than those for Size 1. This is as expected, as Size 2 DGPs include unmodelled serial error dependence which does not appear in Size 1 DGPs. Notice also, that when comparing Table 2 with Table 3, there is a cost to having missing variables in the models specified and used to construct the recursive predictions. Namely, empirical rejection frequencies are generally worse in Table 2 than in Table 3. Overall, though, the recursive block bootstrap appears to handle (missing variable) misspecification quite well, as long as unmodelled error persistence is not too large. Finally, inspection of the last column of each of Tables 2 and 3 reveals that the Kilian parametric bootstrap performs substantially worse than the recursive bootstrap when used to form critical values for the CS test. On the other hand, we shall see that the Kilian bootstrap performs

\footnotetext{
${ }^{21}$ This is confirmed by additional findings using block lengths of 15 and 20, which are available upon request.
} 
very well for the other tests considered in our experiments (see Tables 4 and 5).

Second, notice that the power figures reported in Tables 2 and 3 are as might be expected. Power increases as $a_{2}$ decreases. Additionally, power can be quite poor when the incorrect nonparametric bootstraps are used. However, the Kilian bootstrap generally leads to CS tests with a high level of power; and indeed power under the Kilian bootstrap usually exceeds that achieved under the nonparametric bootstrap, signalling a power-size trade-off when choosing between use of the (asymptotically valid) recursive nonparametric or the parametric bootstrap.

In summary, the recursive bootstrap appears to perform extremely well when compared with the standard nonparametric block bootstrap; and also performs favorably when compared with the parametric Kilian bootstrap, at least with respect to empirical level performance. Additionally, misspecification and dependence clearly play an important role. For example, the Kilian bootstrap is affected more adversely under misspecification (compare the changes in empirical level results when moving from Table 2 to Table 3 for the Kilian bootstrap with those when moving from Table 2 to Table 3 for the recursive bootstrap - the recursive bootstrap is clearly more robust to misspecification in the form of missing variables).

We now turn to a discussion of Tables 4 and 5, where results for the rest of the test statistics examined in our Monte Carlo experiments are reported.

First, we find that the $F$ test is highly oversized. This is due to the poor small sample limit distribution approximation properties of our critical values. Performance would undoubtedly be improved if other limit distribution approximation methods were used, such as asymptotically valid bootstrap methods. For example, in Table 5 (no missing variable in estimated models) the empirical rejection frequencies are around 0.25, even for the least persistent case where $a_{2}=0.3$. Interestingly, the empirical level does not worsen further in Table 6 (missing variable in estimated models), suggesting a robustness of the F-test. However, as the empirical level is so poor anyway, this is not really exciting.

Second, we note that the CM encompassing test performs very well, no matter which critical values are used (i.e. regardless of whether we assume that $\pi=0$ or $\pi>0$ - see footnote to Table 4 for further explanation of $\pi$ ). As might be expected, however, CM test empirical level values are farther from nominal levels in Table 5 than in Table 4. This illustrates the effect of misspecification in the form of missing variables, given that Table 5 has tests constructed using predictions based upon forecasting models where there is a missing variable (i.e. $w_{t-1}$ ). Of note is that we set the 
forecast horizon equal to one-period ahead, and for multiple step ahead prediction, the Kilian bootstrap should dominate the simulated critical values from McCracken (2004) that we use, as discussed in Clark and McCracken (2004)). Further analysis of this issue is left to future research. Thus, though designed for comparing linear models, the CM test seems to have quite good power against nonlinear alternatives.

Third, we see that critical values based upon the Kilian bootstrap perform admirably when used with the DM and the CM tests, but not when used with the CCS tests. When implementing the CCS tests (as was the case with the CS test), the use of the recursive block bootstrap gives rise to tests with rejection rates much closer to the nominal ones. Overall, by jointly comparing Table 3 and 5 , we note that for not too highly dependent observations, the CS test based on the recursive block bootstrap compares adequately with the CM and DM tests based on the Kilian bootstrap.

Fourth, it should be noted that increased lagged endogenous variable dependence has a markedly lesser impact on results in Tables 4 and 5. This result that is not surprising, and underscores well known limitations when using the block bootstrap instead of the parametric bootstraps or (asymptotic) limiting distributions, for forming critical values, when such approaches are available and can be shown to be asymptotically valid.

Finally, note that in the case of Table 4 and 5, power are often unity, suggesting that benchmark misspecification is fairly easy to detect in our experiments. Thus, although our experiments have much to say about the usefulness of the recursive bootstrap, and about the effect on empirical test level of model misspecification, further experiments need to be done in order to better understand power implications of misspecification.

\section{Empirical Illustration}

In this section we implement the F, CM, DM, CS and CCS tests that are described in Table 1, and examined in the previous Monte Carlo section. In particular, we use these tests in order to assess the marginal predictive content of unemployment for core CPI inflation. Recent contributions to this important literature include the papers of Bachmeier and Swanson (2005), Clark and McCracken (2001), Staiger, Stock and Watson (1997), and Stock and Watson (1999). It should be stressed that the results presented in this section are meant primarily to illustrate the uses of the different tests, and to underscore potentially important differences between the tests. Issues of nonlinear model 
selection and structural breaks, for example, are addressed elsewhere.

The data which we use are monthly, and span the period 1955:1-2004:12. The CPI data series is the consumer price index for all urban consumers, all items, seasonally adjusted (Bureau of Labor Statistics series id CPIAUCSL). The unemployment series is the civilian unemployment rate, seasonally adjusted (Bureau of Labor Statistics series id UNRATE).

We construct tests statistics using 1-steap ahead forecasts formed via recursively estimated models. Thus, models are re-estimated (using least squares) at each point in time, before each new prediction is constructed. The beginning date for the in-sample period is 1955:1. The prediction periods reported on are 1970:1-2004:12 and 1984:1-2004:12, so that initial estimation samples include data for the periods 1955:1-1969:12 and 1955:1-1983:12, respectively. The block length is set equal to 6 in application of the recursive block bootstrap. Complete results analogous to those reported in the Monte Carlo section of this paper have been tabulated, and are available upon request. $^{22}$ In all cases, the dependent variable in regressions and the target variable in forecasts is the first log difference of CPI. All models are linear, and explanatory variables include lags of inflation (benchmark or "small" model) and lags of either unemployment or differenced unemployment (alternative model). Lags are selected via use of the Schwarz information criterion. Given this setup, our tests can be viewed as tests of (non) linear Granger causality.

Results are gathered in Tables 6-7. In Tables 6 mean square forecast errors (MSFEs) are tabulated for the "small model" which only contains lags of inflation and the "big model" which contains lags of inflation and lags of unemployment or lags of differenced unemployment. ${ }^{23}$ In reporting MSFEs, additional results are given not only for the two prediction periods outlined above, but also for all periods beginning with 1965:1, 1966:1, ..., 1995:1. Table 7 contains a summary of our findings based on the application of the test statistics discussed above. A number of results emerge upon inspection of the tables. Consider the results in Table 6 .

First, note that the "big" model which uses lags of unemployment (3rd column of the table) yields higher MSFEs than when differenced unemployment is used (4th column of the table) for

\footnotetext{
${ }^{22}$ It should be noted that we do not use real-time data in this empirical illustration, even though both variables considered are subject to periodic revision. Extension of our results to incorporate real-time data is left to future research.

${ }^{23}$ Unemployment appears in our models in both differenced and undifferenced form because there is no consensus on which transformation is appropriate, both from a predictive perspective and from the perspective of valid statistical inference.
} 
all prediction periods beginning 1965:1 until 1975:1. However, when the prediction period begins in 1983:1, the model with differenced unemployment yields a higher MSFE, and this holds for all remaining predictive period begin dates through 1995:1. When predictive period start dates range from 1976:1 through 1982:1, the "low MSFE winner" flip-flops around. Thus, it remains unclear whether unemployment should be differenced or not. This may in part be due to the fact that our linear models can at best be viewed a rough approximations to the true underlying DGP driving the inflation process. Furthermore, as we are only interested in illustrating the application of the recursive block bootstrap, structural breaks are not taken account of.

Second, predictions of inflation have clearly gotten substantially more accurate over our sample period, as evidenced by the fact that MSFEs are much bigger for early subsamples, and are much smaller for the later sub-samples. This result may be in part due to the smooth nature of recent data relative to more distant data, although it is difficult to say with certainty what is causing this feature of our models.

Finally, there is clearly very little evidence of predictive power of unemployment for inflation, as evidenced by the fact that entries for the "small" model that does not contain lags of unemployment (column 2 of the table) are always very close to entries in the remaining columns of entries in the table, where unemployment lags are added to the model. This evidence is in loose agreement with the results reported by Clark and McCracken (2001), where it was found that in-sample evidence of Granger causality was much stronger than out-of-sample evidence. It should be stressed, however, that thus far we have only compared MSFEs, and hence have focused our attention upon the comparison of purely linear models. In order to assess the potential impact of generic nonlinearity, we need to either fit a variety of nonlinear models (which may be a large undertaking, given the plethora of available models), or we need to carry out tests such as the generically comprehensive nonlinear Granger causality CS test. We turn to this issue next. In particular, please refer to Table 7 for the remainder of our discussion. A number of findings emerge.

First, the CS and CCS test result in the only rejections of the non-causality null hypothesis. This suggests that the CS and CCS tests are capturing some feature of the data not captured by the other tests. Since the CS test is designed to have generic power against nonlinear alternatives while all of the other tests are designed to have power against linear alternatives, one possibility is that we have evidence of nonlinear Granger causality. Furthermore, since the other tests always fail to reject, we have evidence against linear Granger causality. Thus, it appears that misspecification 
in the form of neglected nonlinearity may be driving our results. Namely, unemployment may have marginal predictive power for inflation, if the right nonlinear model were to be specified. However, we have included two different prediction period start dates in order to illustrate that findings may change depending upon the sample of data being examined. In particular, there are almost no rejections at all when the prediction period begins in 1984:1. This suggests that the type of nonlinear misspecification driving our results for the longer prediction period may actually be the presence of structural breaks. It is difficult to say anything more concrete in this regard, without carrying out a complete and comprehensive examination of the data, and this is left to future research. Suffice it to say that the different tests examined may yield different conclusions, as might be expected, given that the CS test is the only one designed specifically to have power against generic nonlinear alternatives.

Second, the in-sample F-test also never rejects, in opposition to the evidence in the literature (see e.g. Clark and McCracken (2001) and Inoue and Kilian (2004)). This result is not too surprising, given our Monte Carlo finding that this test is oversized in finite samples. However, it should be noted that application of different sampling strategies (e.g. the use of rolling windows), and examination of more prediction periods does lead to some rejection using the F-test, in accord with the results of Clark and McCracken (2001) and Inoue and Kilian (2004). These additional finding are summarized in an earlier working paper version of this paper, but are left out of the current paper for the sake of brevity.

Third, it is interesting to note that the CS and CCS tests tended to have lower power than the CM and DM tests, in our Monte Carlo experiments. Additionally, for block lengths similar to those considered in this empirical illustration, the CS test was shown to be conservative. In stark contrast to these experimental findings, our empirical results suggest that only the CS and CCS seem to be able to reject the null hypothesis, lending further evidence of neglected nonlinearity.

In summary, much empirical work is needed before a complete picture emerges concerning the prevalence of nonlinear Granger causality in the unemployment/inflation relationship. This is left to future research. It is clear, however, that much can be learned by using all of the different tests in consort with one another. The picture that emerges when only a subset of the tests is used to analyze the marginal predictive content of unemployment for inflation is that of an absence of predictive ability. When all of the tests are used, on the other hand, interesting evidence arises concerning the potential nonlinear predictive content of unemployment. Thus, the tests discussed 
in this illustration appear to be useful complements to each other.

\section{Conclusions}

In many instances, test statistics based on recursive and/or rolling estimation schemes have limiting distributions which are functionals of Gaussian processes, and which have covariance kernels that reflect parameter uncertainty. In these cases, limiting distributions are thus not nuisance parameter free, and valid critical values are often obtained via bootstrap methods. In this paper, we first developed a bootstrap procedure that properly captures the contribution of parameter estimation error in recursive estimation schemes using dependent data. Intuitively, when parameters are estimated recursively, as is done in our framework, earlier observations in the sample enter into test statistics more frequently than later observations. This induces a location bias in the bootstrap distribution, which can be either positive or negative across different samples, and hence the bootstrap modification that we discuss is required in order to obtain first order validity of the bootstrap. Within this framework, we then presented two applications, both based on forecasting tests. In particular, our applications extend the White (2000) reality check to the case of non vanishing parameter estimation error, and extend the integrated conditional moment (ICM) tests of Bierens (1982, 1990) and Bierens and Ploberger (1997) to the case of out-of-sample prediction. Of note is that in both of these examples, it is shown that we must construct bootstrap statistics that are different from the "usual" bootstrap statistics, which are defined as the difference between the statistic computed over the sample observations and over the bootstrap observations. This feature of our applications suggests that one must be careful when forming bootstrap statistics in all cases for which recursive estimation is used and predictive model selection is the objective. Finally, results of a Monte Carlo investigation of a variety of related tests, and an empirical illustration are presented. 


\section{Appendix}

As the statements below hold for $i=1, \ldots, n$, and given that the proofs are the same regardless which model is considered, for notational simplicity we drop the subscript $i$.

Proof of Theorem 1: Given (7), by first order conditions,

$$
\frac{1}{t} \sum_{j=s}^{t}\left(\nabla_{\theta} q\left(y_{j}^{*}, Z^{*, j-1}, \widetilde{\theta}_{t}^{*}\right)-\left(\frac{1}{T} \sum_{k=s}^{T-1} \nabla_{\theta} q\left(y_{k}, Z^{k-1}, \widehat{\theta}_{t}\right)\right)\right)=0 .
$$

Thus, a Taylor expansion around $\widehat{\theta}_{t}$ yields:

$$
\begin{aligned}
\left(\widetilde{\theta}_{t}^{*}-\widehat{\theta}_{t}\right)= & \left(-\frac{1}{t} \sum_{j=s}^{t} \nabla_{\theta}^{2} q\left(y_{j}^{*}, Z^{*, j-1}, \bar{\theta}_{t}^{*}\right)\right)^{-1} \\
& \times\left(\frac{1}{t} \sum_{j=s}^{t}\left(\nabla_{\theta} q\left(y_{j}^{*}, Z^{*, j-1}, \widehat{\theta}_{t}\right)-\left(\frac{1}{T} \sum_{k=s}^{T-1} \nabla_{\theta} q\left(y_{k}, Z^{k-1}, \widehat{\theta}_{t}\right)\right)\right),\right.
\end{aligned}
$$

where $\bar{\theta}_{t}^{*} \in\left(\widetilde{\theta}_{t}^{*}, \widehat{\theta}_{t}\right)$. Hereafter, let $B^{\dagger}=\left(E\left(-\nabla_{\theta}^{2} q\left(y_{j}, Z^{j-1}, \theta^{\dagger}\right)\right)\right)^{-1}$. Recalling that we resample from the entire sample, regardless the value of $t$, it follows that:

$$
\frac{1}{t} \sum_{j=s}^{t} E^{*}\left(\nabla_{\theta}^{2} q\left(y_{j}^{*}, Z^{*, j-1}, \theta\right)\right)=\frac{1}{T} \sum_{k=s}^{T-1} \nabla_{\theta}^{2} q\left(y_{k}, Z^{k-1}, \theta\right)+O_{P^{*}}\left(\frac{l}{T}\right), \operatorname{Pr}-P,
$$

where the $O_{P^{*}}\left(\frac{l}{T}\right)$ term is due to the end effect (i.e. due to the contribution of the first and last $l$ observations, as shown in Lemma A1 in Fitzenberger (1997)). Thus,

$$
\begin{aligned}
& \operatorname{supsup}_{t \geq R}\left|\left(-\frac{1}{t} \sum_{j=\Theta}^{t} \nabla_{\theta}^{2} q\left(y_{j}^{*}, Z^{*, j-1}, \theta\right)\right)^{-1}-B^{\dagger}\right| \\
\leq & \operatorname{supsup}_{t \geq R}\left|\left(-\frac{1}{t} \sum_{j \in \Theta}^{t} \nabla_{\theta}^{2} q\left(y_{j}^{*}, Z^{*, j-1}, \theta\right)\right)^{-1}-\left(\frac{1}{t} \sum_{j=s}^{t} E^{*}\left(-\nabla_{\theta}^{2} q\left(y_{j}^{*}, Z^{*, j-1}, \theta\right)\right)\right)^{-1}\right| \\
+ & \operatorname{supsup}_{t \geq R}\left|\left(\frac{1}{t} \sum_{j \in \Theta}^{t} E^{*}\left(-\nabla_{\theta}^{2} q\left(y_{j}^{*}, Z^{*, j-1}, \theta\right)\right)\right)^{-1}-B^{\dagger}\right| .
\end{aligned}
$$

Given (20), and Assumptions A1-A2, the second term on the RHS of (21) is $o_{P}(1)$. Recalling also that the resampled series consists of $b$ independent and identically distributed blocks, and that $b / T^{1 / 2} \rightarrow \infty$, it follows that the first term on on the RHS of (21) is $o_{P^{*}}(1) \operatorname{Pr}-P$, given the uniform 
law of large number for iid random variables. Thus,

$$
\begin{aligned}
& \frac{1}{\sqrt{P}} \sum_{t=R}^{T-1}\left(\widetilde{\theta}_{t}^{*}-\widehat{\theta}_{t}\right) \\
= & B^{\dagger} \frac{1}{\sqrt{P}} \sum_{t=R}^{T-1}\left(\frac{1}{t} \sum_{j=s}^{t}\left(\nabla_{\theta} q\left(y_{j}^{*}, Z^{*, j-1}, \widehat{\theta}_{t}\right)-\left(\frac{1}{T} \sum_{k=s}^{T-1} \nabla_{\theta} q\left(y_{k}, Z^{k-1}, \widehat{\theta}_{t}\right)\right)\right)\right) \\
& +o_{P^{*}}(1) \operatorname{Pr}-P,
\end{aligned}
$$

and a first order expansion of the RHS of (22) around $\theta^{\dagger}$ yields:

$$
\begin{aligned}
& \frac{1}{\sqrt{P}} \sum_{t=R}^{T-1}\left(\widetilde{\theta}_{t}^{*}-\widehat{\theta}_{t}\right) \\
= & B^{\dagger} \frac{1}{\sqrt{P}} \sum_{t=R}^{T-1}\left(\frac{1}{t} \sum_{j=s}^{t}\left(\nabla_{\theta} q\left(y_{j}^{*}, Z^{*, j-1}, \theta^{\dagger}\right)-\left(\frac{1}{T} \sum_{k=s}^{T-1} \nabla_{\theta} q\left(y_{k}, Z^{k-1}, \theta^{\dagger}\right)\right)\right)\right) \\
& +B^{\dagger} \frac{1}{\sqrt{P}} \sum_{t=R}^{T-1}\left(\left(\frac{1}{t} \sum_{j=s}^{t}\left(\nabla_{\theta}^{2} q\left(y_{j}^{*}, Z^{*, j-1}, \bar{\theta}_{t}\right)-\left(\frac{1}{T} \sum_{k=s}^{T-1} \nabla_{\theta}^{2} q\left(y_{k}, Z^{k-1}, \bar{\theta}_{t}\right)\right)\right)\right)\right. \\
& \left.\times\left(\widehat{\theta}_{t}-\theta^{\dagger}\right)\right)+o_{P^{*}}(1) \operatorname{Pr}-P .
\end{aligned}
$$

We need to show that the second term on the RHS of (23) is $o_{P^{*}}(1) \operatorname{Pr}-P$. Note that this term is majorized by

$$
B^{\dagger} \sup _{t \geq R} \sup _{\theta \in \Theta} \frac{\sqrt{P}}{t^{1+\vartheta}}\left|\sum_{j=s}^{t}\left(\nabla_{\theta}^{2} q\left(y_{j}^{*}, Z^{*, j-1}, \theta\right)-\left(\frac{1}{T} \sum_{k=s}^{T-1} \nabla_{\theta}^{2} q\left(y_{k}, Z^{k-1}, \theta\right)\right)\right)\right| \sup _{t \geq R} t^{\vartheta}\left|\widehat{\theta}_{t}-\theta^{\dagger}\right|
$$

with $1 / 3<\vartheta<1 / 2$. Recalling also that $b l=T$ and $l=o\left(T^{1 / 4}\right)$, it follows that $b / T^{3 / 4} \rightarrow \infty$. Thus, by the same argument used in Lemma 1(i) in Altissimo and Corradi (2002), and given (20), it follows that:

$$
\sup _{t \geq R}\left|\frac{1}{\theta} \sum_{\theta \in \Theta}^{t}\left(\nabla_{\theta}^{2} q\left(y_{j}^{*}, Z^{*, j-1}, \theta\right)-\left(\frac{1}{T} \sum_{k=s}^{T-1} \nabla_{\theta}^{2} q\left(y_{k}, Z^{k-1}, \theta\right)\right)\right)\right|=O_{\text {a.s. }}\left(\sqrt{\frac{\log \log b}{b}}\right), \text { a.s. }-P .
$$

Thus,

$$
\sup _{t \geq R} \sup _{\theta \in \Theta} \frac{\sqrt{P}}{t^{1+\vartheta}}\left|\sum_{j=s}^{t}\left(\nabla_{\theta}^{2} q\left(y_{j}^{*}, Z^{*, j-1}, \theta\right)-\left(\frac{1}{T} \sum_{j=s}^{T} \nabla_{\theta}^{2} q\left(y_{j}, Z^{j-1}, \theta\right)\right)\right)\right|=o_{P^{*}}(1), \operatorname{Pr}-P
$$


for $\vartheta>1 / 3$. Finally, for all $\vartheta<1 / 2, \sup _{t \geq R} t^{\vartheta}\left|\widehat{\theta}_{t}-\theta^{\dagger}\right|=o_{P}(1)$ by Lemma A3 in West (1996). Recalling that

$$
\frac{1}{t} \sum_{j=s}^{t} E^{*}\left(\nabla_{\theta} q\left(y_{j}^{*}, Z^{*, j-1}, \theta^{\dagger}\right)\right)=\frac{1}{T} \sum_{k=s}^{T-1} \nabla_{\theta} q\left(y_{k}, Z^{k-1}, \theta^{\dagger}\right)+O_{P}\left(\frac{l}{T}\right),
$$

the right hand side of (23) can be written as:

$$
\begin{aligned}
& \frac{1}{\sqrt{P}} \sum_{t=R}^{T-1}\left(\widetilde{\theta}_{t}^{*}-\widehat{\theta}_{t}\right) \\
= & B^{\dagger} \frac{1}{\sqrt{P}} \sum_{t=R}^{T-1}\left(\frac{1}{t} \sum_{j=s}^{t}\left(\nabla_{\theta} q\left(y_{j}^{*}, Z^{*, j-1}, \theta^{\dagger}\right)-E^{*}\left(\nabla_{\theta} q\left(y_{j}^{*}, Z^{*, j-1}, \theta^{\dagger}\right)\right)\right)+o_{P^{*}}(1) \operatorname{Pr}-P\right. \\
= & B^{\dagger} \frac{a_{R, 0}}{\sqrt{P}} \sum_{j=1}^{R}\left(\nabla_{\theta} q\left(y_{j}^{*}, Z^{*, j-1}, \theta^{\dagger}\right)-E^{*}\left(\nabla_{\theta} q\left(y_{j}^{*}, Z^{*, j-1}, \theta^{\dagger}\right)\right)\right) \\
& +B^{\dagger} \frac{1}{\sqrt{P}} \sum_{j=1}^{P-1} a_{R, j}\left(\nabla_{\theta} q\left(y_{R+j}^{*}, Z^{*, R+j-1}, \theta^{\dagger}\right)-E^{*}\left(\nabla_{\theta} q\left(y_{R+j}^{*}, Z^{*, R+j-1}, \theta^{\dagger}\right)\right)\right) \\
& +o_{P^{*}}(1) \operatorname{Pr}-P,
\end{aligned}
$$

where $a_{R, i}=(R+i)^{-1}+\ldots+(R+P-1)^{-1}$, for $0 \leq i<P-1$. The second equality on the RHS of (24) follows directly from Lemma A5 in West (1996).

Additionally the first two terms on the RHS of (24) satisfy a central limit theorem for triangular independent arrays (see e.g. White and Wooldridge (1988)); and thus, conditional on the sample, $\frac{1}{\sqrt{P}} \sum_{t=R}^{T-1}\left(\widetilde{\theta}_{t}^{*}-\widehat{\theta}_{t}\right)$ converges in distribution to a zero mean normal random variable.

Furthermore, by Theorem 4.1 in West (1996):

$$
\frac{1}{\sqrt{P}} \sum_{t=R}^{T}\left(\widehat{\theta}_{t}-\theta^{\dagger}\right) \stackrel{d}{\rightarrow} N\left(0,2 \Pi B^{\dagger} C_{00} B^{\dagger}\right),
$$

where $C_{00}=\sum_{j=-\infty}^{\infty} E\left(\left(\nabla_{\theta} q\left(y_{1+s}, Z^{s}, \theta^{\dagger}\right)\right)\left(\nabla_{\theta} q\left(y_{1+s+j}, Z^{s+j}, \theta^{\dagger}\right)\right)^{\prime}\right)$ and $\Pi=1-\pi^{-1} \ln (1+\pi)$. Therefore, the statement in the theorem will follow once we have shown that:

$$
\operatorname{Var}^{*}\left(\frac{1}{\sqrt{P}} \sum_{t=R}^{T}\left(\widetilde{\theta}_{t}^{*}-\widehat{\theta}_{t}\right)\right)=2 \Pi B^{\dagger} C_{00} B^{\dagger}, \operatorname{Pr}-P .
$$

For notational simplicity, let $\nabla_{\theta} q\left(y_{j}^{*}, Z^{*, j-1}, \theta^{\dagger}\right)=h_{j}^{*}$, and let $\nabla_{\theta} q\left(y_{j}, Z^{j-1}, \theta^{\dagger}\right)=h_{j}$. Then, given (24):

$$
\operatorname{Var}^{*}\left(\frac{1}{\sqrt{P}} \sum_{t=R}^{T-1} h_{t}^{*}\right)=\frac{R}{P} \operatorname{Var}^{*}\left(a_{R, 0} \frac{1}{\sqrt{R}} \sum_{j=1}^{R} h_{j}^{*}\right)
$$




$$
+\frac{1}{P} \operatorname{Var}^{*}\left(\sum_{j=1}^{P-1} a_{R, j} h_{R+j}^{*}\right)+\frac{1}{P} \operatorname{Cov}^{*}\left(a_{R, 0} \sum_{j=1}^{R} h_{j}^{*}, \sum_{j=1}^{P-1} a_{R, j} h_{R+j}^{*}\right) .
$$

As all blocks are independent, conditional on the sample, the covariance term in this expression is equal to zero. Without loss of generality, set $R=b_{1} l$ and $P=b_{2} l$, where $b_{1}+b_{2}=b$. It then follows that, up to a term of order $O\left(l / R^{1 / 2}\right)$,

$$
\begin{gathered}
\operatorname{Var}^{*}\left(a_{R, 0} \frac{1}{\sqrt{R}} \sum_{j=1}^{R} h_{j}^{*}\right)=a_{R, 0}^{2} \operatorname{Var}^{*}\left(\frac{1}{\sqrt{R}} \sum_{k=1}^{b_{1}} \sum_{i=1}^{l} h_{I_{k}+i}\right) \\
=a_{R, 0}^{2} E^{*}\left(\frac{1}{R} \sum_{k=1}^{b_{1}} \sum_{i=1}^{l} \sum_{k=1}^{l}\left(h_{I_{k}+i}-\bar{h}_{T}\right)\left(h_{I_{k}+j}-\bar{h}_{T}\right)^{\prime}\right) \\
=a_{R, 0}^{2}\left(\frac{1}{R} \sum_{t=l}^{R-l} \sum_{j=-l}^{l}\left(h_{t}-\bar{h}_{T}\right)\left(h_{t+j}-\bar{h}_{T}\right)^{\prime}\right)+O\left(l / R^{1 / 2}\right) \operatorname{Pr}-P,
\end{gathered}
$$

where $\bar{h}_{T}=\frac{1}{T} \sum_{t=s}^{T} h_{t}$. Thus,

$$
\begin{aligned}
& \frac{R}{P} \operatorname{Var}^{*}\left(a_{R, 0} \frac{1}{\sqrt{R}} \sum_{j=1}^{R} h_{j}^{*}\right) \\
= & \frac{R a_{R, 0}^{2}}{P} \sum_{j=-l}^{l} \gamma_{j}+\frac{R a_{R, 0}^{2}}{P}\left(\frac{1}{R} \sum_{t=l}^{R-l} \sum_{j=-l}^{l}\left(\left(h_{t}-\bar{h}_{T}\right)\left(h_{t+j}-\bar{h}_{T}\right)^{\prime}-\gamma_{j}\right)\right)+O\left(\frac{l^{2}}{R}\right),
\end{aligned}
$$

where $\gamma_{j}=\operatorname{Cov}\left(h_{1}, h_{1+j}\right)$. By West $\left(1996\right.$, proof of Lemma A5), it follows that $\frac{R a_{R, 0}^{2}}{P} \sum_{j=-l}^{l} \gamma_{j} \rightarrow$ $\pi^{-1} \ln ^{2}(1+\pi) C_{00}$, while the second term on the RHS above goes to zero, $\operatorname{Pr}-P$ (see e.g. Theorem 2 in Newey and West (1987)). Now, up to a term of order $O\left(l / P^{1 / 2}\right) \operatorname{Pr}-P$ :

$$
\begin{aligned}
& \operatorname{Var}^{*}\left(\frac{1}{\sqrt{P}} \sum_{j=1}^{P-1} a_{R, j} h_{R+j}^{*}\right)=\operatorname{Var}^{*}\left(\frac{1}{\sqrt{P}} \sum_{k=b_{1}+1}^{b} \sum_{i=1}^{l} a_{R,((k-1) l+i)} h_{I_{k}+i}\right) \\
= & \frac{1}{P} E^{*}\left(\sum_{k=b_{1}+1}^{b} \sum_{i=1}^{l} \sum_{j=1}^{l} a_{R,\left(\left(k-b_{1}-1\right) l+i\right)} a_{R,\left(\left(k-b_{1}-1\right) l+j\right)}\left(h_{I_{k}+i}-\bar{h}_{T}\right)\left(h_{I_{k}+j}-\bar{h}_{T}\right)^{\prime}\right) \\
= & \frac{1}{P} \sum_{k=b_{1}+1}^{b} \sum_{i=1}^{l} \sum_{j=1}^{l} a_{R,\left(\left(k-b_{1}-1\right) l+i\right)} a_{R,\left(\left(k-b_{1}-1\right) l+j\right)} E^{*}\left(\left(h_{I_{k}+i}-\bar{h}_{T}\right)\left(h_{I_{k}+j}-\bar{h}_{T}\right)^{\prime}\right)
\end{aligned}
$$




$$
\begin{aligned}
= & \frac{1}{P} \sum_{k=b_{1}+1}^{b} \sum_{i=1}^{l} \sum_{j=1}^{l} a_{R,\left(\left(k-b_{1}-1\right) l+i\right)} a_{R,\left(\left(k-b_{1}-1\right) l+j\right)}\left(\frac{1}{T} \sum_{t=l}^{T-l}\left(h_{t+i}-\bar{h}_{P}\right)\left(h_{+t+j}-\bar{h}_{P}\right)^{\prime}\right)+O\left(l / P^{1 / 2}\right) \operatorname{Pr}-P \\
= & \frac{1}{P} \sum_{k=b_{1}+1}^{b} \sum_{i=1}^{l} \sum_{j=1}^{l} a_{R,\left(\left(k-b_{1}-1\right) l+i\right)} a_{R,\left(\left(k-b_{1}-1\right) l+j\right)} \gamma_{i-j} \\
& +\frac{1}{P} \sum_{k=b_{1}+1}^{b} \sum_{i=1}^{l} \sum_{j=1}^{l} a_{R,\left(\left(k-b_{1}-1\right) l+i\right)} a_{R,\left(\left(k-b_{1}-1\right) l+j\right)}\left(\frac{1}{T} \sum_{t=l}^{T-l}\left(\left(h_{t+i}-\bar{h}_{T}\right)\left(h_{t+j}-\bar{h}_{T}\right)^{\prime}-\gamma_{i-j}\right)\right) \\
& +O\left(l / P^{1 / 2}\right) \operatorname{Pr}-P
\end{aligned}
$$

We need to show that the last term on the last equality in (27) is $o(1) \operatorname{Pr}-P$. First note that this term is majorized by

$$
\begin{aligned}
& \left|\frac{b_{2}}{P} \sum_{i=1}^{l} \sum_{j=1}^{l}\left(\frac{1}{T} \sum_{t=l}^{T-l}\left(\left(h_{t+i}-\bar{h}_{T}\right)\left(h_{t+j}-\bar{h}_{T}\right)^{\prime}-\gamma_{i-j}\right)\right)\right| \\
= & \left|\frac{1}{T} \sum_{t=l}^{T-l} \sum_{j=-l}^{l}\left(\left(h_{t}-\bar{h}_{T}\right)\left(h_{t+j}-\bar{h}_{T}\right)^{\prime}-\gamma_{j}\right)\right|+O\left(l / P^{1 / 2}\right) \operatorname{Pr}-P .
\end{aligned}
$$

The first term on the RHS of (28) goes to zero in probability, by the same argument as that used in Lemma 2 in Corradi (1999). ${ }^{24}$ With regard to the first term on the RHS of the last equality in (27), note that:

$$
\begin{gathered}
\frac{1}{P} \sum_{k=1}^{b_{2}} \sum_{i=1}^{l} \sum_{j=1}^{l} a_{R,((k-1) l+i)} a_{R,((k-1) l+j)} \gamma_{i-j}=\frac{1}{P} \sum_{t=l}^{P-l} \sum_{j=-l}^{l} a_{R, t} a_{R, t+j} \gamma_{j}+O\left(l / P^{1 / 2}\right) \operatorname{Pr}-P \\
=\frac{1}{P} \sum_{t=l}^{P-l} a_{R, t}^{2} \sum_{j=-l}^{l} \gamma_{j}+\frac{1}{P} \sum_{t=l}^{P-l} \sum_{j=-l}^{l}\left(a_{R, t} a_{R, t+j}-a_{R, t}^{2}\right) \gamma_{j}+O\left(l / P^{1 / 2}\right) \operatorname{Pr}-P .
\end{gathered}
$$

By the same argument as that used in Lemma A5 of West (1996), the second term on the RHS above approaches zero, while:

$$
\frac{1}{T} \sum_{t=l}^{P-l} a_{R, t}^{2} \sum_{j=-l}^{l} \gamma_{j} \rightarrow\left(2\left[1-\pi^{-1} \ln (1+\pi)\right]-\pi^{-1} \ln ^{2}(1+\pi)\right) C_{00}
$$

As the first term on the RHS of (26) converges to $\pi^{-1} \ln ^{2}(1+\pi) C_{00}$ (see West (1996), p.1082), the desired outcome then follows.

\footnotetext{
${ }^{24}$ The domination conditions here are weaker than those in Lemma 2 in Corradi (1999), as we require only convergence to zero in probability, and not almot sure convergence.
} 
Proof of Proposition 1: Let $\bar{u}_{i, t}=y_{t}-\kappa\left(Z^{t-1}, \bar{\theta}_{i, t}\right)$, with $\bar{\theta}_{i, t} \in\left(\widehat{\theta}_{i, t}, \theta^{\dagger}\right)$. Via a mean value expansion, and given Assumptions A1-A2:

$$
\begin{aligned}
S_{P}(1, k)= & \frac{1}{P^{1 / 2}} \sum_{t=R}^{T-1}\left(g\left(\widehat{u}_{1, t+1}\right)-g\left(\widehat{u}_{k, t+1}\right)\right) \\
= & \frac{1}{P^{1 / 2}} \sum_{t=R}^{T-1}\left(g\left(u_{1, t+1}\right)-g\left(u_{k, t+1}\right)\right) \\
& +\frac{1}{P} \sum_{t=R}^{T-1} g^{\prime}\left(\bar{u}_{1, t+1}\right) \nabla_{\theta_{1}} \kappa_{1}\left(Z^{t}, \bar{\theta}_{1, t}\right) P^{1 / 2}\left(\widehat{\theta}_{1, t}-\theta_{1}^{\dagger}\right) \\
& -\frac{1}{P} \sum_{t=R}^{T-1} g^{\prime}\left(\bar{u}_{k, t+1}\right) \nabla_{\theta_{k}} \kappa_{k}\left(Z^{t}, \bar{\theta}_{k, t}\right) P^{1 / 2}\left(\widehat{\theta}_{k, t}-\theta_{k}^{\dagger}\right) \\
= & \frac{1}{P^{1 / 2}} \sum_{t=R}^{T-1}\left(g\left(u_{1, t+1}\right)-g\left(u_{k, t+1}\right)\right) \\
& +\mu_{1} \frac{1}{P^{1 / 2}} \sum_{t=R}^{T-1}\left(\widehat{\theta}_{1, t}-\theta_{1}^{\dagger}\right)-\mu_{k} \frac{1}{P^{1 / 2}} \sum_{t=R}^{T-1}\left(\widehat{\theta}_{k, t}-\theta_{k}^{\dagger}\right)+o_{P}(1),
\end{aligned}
$$

where $\mu_{1}=E\left(g^{\prime}\left(u_{1, t+1}\right) \nabla_{\theta_{1}} \kappa_{1}\left(Z^{t}, \theta_{1}^{\dagger}\right)\right)$, and $\mu_{k}$ is defined analogously. Now, when all competitors have the same predictive accuracy as the benchmark model, by the same argument as that used in Theorem 4.1 of West (1996), it follows that:

$$
\left(S_{P}^{\mu}(1,2), \ldots, S_{P}^{\mu}(1, n)\right) \stackrel{d}{\rightarrow} N(0, V)
$$

where $S_{P}^{\mu}(1, k)=S_{P}(1, k)-\sqrt{P} E\left(g\left(u_{1, t+1}\right)-g\left(u_{k, t+1}\right)\right)$, and where $V$ is an $n \times n$ matrix with $i, j$ element $v_{i, j}$ defined in the statement of the proposition. The distribution of $S_{P}$ then follows as a straightforward application of the continuous mapping theorem. $\square$

Proof of Theorem 2: Let $\widehat{u}_{i, t+1}^{*}=y_{t+1}^{*}-\kappa_{i}\left(Z^{*, t}, \widehat{\theta}_{i, t}\right), \bar{u}_{i, t+1}^{*}=y_{t+1}^{*}-\kappa_{i}\left(Z^{*, t}, \bar{\theta}_{i, t}^{*}\right)$, with $\bar{\theta}_{i, t}^{*} \in$ $\left(\widetilde{\theta}_{i, t}^{*}, \widehat{\theta}_{i, t}\right)$, Additionally, let $\widehat{u}_{i, j+1}^{(t)}=y_{j+1}-\kappa_{i}\left(Z^{j}, \widehat{\theta}_{i, t}\right)$. It follows that:

$$
\begin{aligned}
S_{P}^{*}(1, k)= & \frac{1}{\sqrt{P}} \sum_{t=R}^{T-1}\left(\left(g\left(\widetilde{u}_{1, t+1}^{*}\right)-g\left(\widetilde{u}_{k, t+1}^{*}\right)\right)-\frac{1}{T} \sum_{j=s}^{T}\left(g\left(\widehat{u}_{1, j+1}^{(t)}\right)-g\left(\widehat{u}_{k, j+1}^{(t)}\right)\right)\right) \\
= & \frac{1}{\sqrt{P}} \sum_{t=R}^{T-1}\left(\left(g\left(\widehat{u}_{1, t+1}^{*}\right)-g\left(\widehat{u}_{k, t+1}^{*}\right)\right)-\frac{1}{T} \sum_{j=s}^{T}\left(g\left(\widehat{u}_{1, j+1}^{(t)}\right)-g\left(\widehat{u}_{k, j+1}^{(t)}\right)\right)\right) \\
& +\frac{1}{P^{1 / 2}} \sum_{t=R}^{T-1}\left(\nabla_{\theta_{1}} g\left(\bar{u}_{1, t+1}^{*}\right)\left(\widehat{\theta}_{1, t}^{*}-\widehat{\theta}_{1, t}\right)-\nabla_{\theta_{1}} g\left(\bar{u}_{k, t+1}^{*}\right)\left(\widehat{\theta}_{k, t}^{*}-\widehat{\theta}_{k, t}\right)\right) .
\end{aligned}
$$


Now,

$$
E^{*}\left(g\left(\widehat{u}_{1, t+1}^{*}\right)-g\left(\widehat{u}_{k, t+1}^{*}\right)\right)=\frac{1}{T} \sum_{j=s}^{T}\left(g\left(\widehat{u}_{1, j+1}^{(t)}\right)-g\left(\widehat{u}_{k, j+1}^{(t)}\right)\right)+O\left(\frac{l}{T}\right) .
$$

Thus, by Theorem 3.5 in Künsch (1989), the first term on the second equality on the RHS of (29) converges in $P^{*}$-distribution to a zero mean normal random variable with variance equal to $\lim _{P \rightarrow \infty} \operatorname{Var}^{*}\left(\frac{1}{\sqrt{P}} \sum_{t=R}^{T-1}\left(g\left(\widehat{u}_{1, t+1}^{*}\right)-g\left(\widehat{u}_{k, t+1}^{*}\right)\right)\right)$, conditional on the sample and for all samples except a subset with probability measure approaching zero. Now, by the same argument used in the proof of Theorem 1:

$$
\operatorname{Var}^{*}\left(\frac{1}{\sqrt{P}} \sum_{t=R}^{T-1}\left(g\left(\widehat{u}_{1, t+1}^{*}\right)-g\left(\widehat{u}_{k, t+1}^{*}\right)\right)\right)=\operatorname{Var}\left(\frac{1}{\sqrt{P}} \sum_{t=R}^{T-1}\left(g\left(\widehat{u}_{1, t+1}\right)-g\left(\widehat{u}_{k, t+1}\right)\right)\right)+o(1) \operatorname{Pr}-P .
$$

This implies that the first term in the second equality on the RHS of (29) has the same limiting distribution as $\frac{1}{\sqrt{P}} \sum_{t=R}^{T-1}\left(\left(g\left(u_{1, t+1}\right)-g\left(u_{k, t+1}\right)\right)-E\left(g\left(u_{1, t+1}\right)-g\left(u_{k, t+1}\right)\right)\right)$, conditional on the sample, and for all samples except a subset with probability measure approaching zero. Finally, the last term in (29) has the same limiting distribution as $\mu_{1} \frac{1}{P^{1 / 2}} \sum_{t=R}^{T-1}\left(\widehat{\theta}_{1, t}^{*}-\widehat{\theta}_{1, t}\right)-$ $\mu_{k} \frac{1}{P^{1 / 2}} \sum_{t=R}^{T-1}\left(\widehat{\theta}_{k, t}^{*}-\widehat{\theta}_{k, t}\right)$, conditional on the sample, and for all samples except a subset with probability measure approaching zero. The statement in the proposition then follows as a straightforward application of the continuous mapping theorem.

Proof of Proposition 2: The proof follows directly from Theorem 1 in Corradi and Swanson (2002).

Proof of Theorem 3: Recall that $g=q_{1}$. Additionally, let $\widetilde{u}_{1, t+1}^{*}=y_{t+1}^{*}-\left(\begin{array}{ll}1 & y_{t}^{*}\end{array}\right) \widetilde{\theta}_{1, t}^{*}, \widehat{u}_{1, t+1}^{*}=$ $y_{t+1}^{*}-\left(\begin{array}{ll}1 & y_{t}^{*}\end{array}\right) \widehat{\theta}_{1, t}, \bar{u}_{1, t+1}^{*}=y_{t+1}^{*}-\left(\begin{array}{ll}1 & y_{t}^{*}\end{array}\right) \bar{\theta}_{1, t}^{*}$, and $\widehat{u}_{1, j+1}^{(t)}=y_{j+1}-\left(\begin{array}{ll}1 & y_{t}\end{array}\right) \widehat{\theta}_{1, t}$, where $\bar{\theta}_{1, t}^{*} \in\left(\widetilde{\theta}_{1, t}^{*}, \widehat{\theta}_{1, t}\right)$. It then follows that:

$$
\begin{aligned}
& \frac{1}{P^{1 / 2}} \sum_{t=R}^{T-1}\left(g^{\prime}\left(\widetilde{u}_{1, t+1}^{*}\right) w\left(Z^{*, t}, \gamma\right)-\frac{1}{T} \sum_{j=2}^{T-1} g^{\prime}\left(\widehat{u}_{1, j+1}^{(t)}\right) w\left(Z^{j}, \gamma\right)\right) \\
= & \frac{1}{P^{1 / 2}} \sum_{t=R}^{T-1}\left(g^{\prime}\left(\widehat{u}_{1, t+1}^{*}\right) w\left(Z^{*, t}, \gamma\right)-\frac{1}{T} \sum_{j=2}^{T-1} g^{\prime}\left(\widehat{u}_{1, j+1}^{(t)}\right) w\left(Z^{j}, \gamma\right)\right) \\
& +\frac{1}{P^{1 / 2}} \sum_{t=R}^{T-1}\left(\nabla_{\theta} g^{\prime}\left(\bar{u}_{1, t+1}^{*}\right) w\left(Z^{*, t}, \gamma\right)\right)\left(\widetilde{\theta}_{1, t}^{*}-\widehat{\theta}_{1, t}\right) .
\end{aligned}
$$

First, note that the first term on the RHS of the last equality in (30) has the same limiting distribution as 
$\frac{1}{P^{1 / 2}} \sum_{t=R}^{T-1}\left(g^{\prime}\left(u_{1, t+1}\right) w\left(Z^{t}, \gamma\right)-E\left(g^{\prime}\left(u_{1, t+1}\right) w\left(Z^{t}, \gamma\right)\right)\right)$, pointwise in $\gamma$. Further, note that stochastic equicontinuity on $\Gamma$ can be shown using the same approach as that used in the proof of Theorem 2 in Corradi and Swanson (2002). Therefore, under $H_{0}$, any continuous functional over $\Gamma$ of $\frac{1}{P^{1 / 2}} \sum_{t=R}^{T-1}\left(g^{\prime}\left(u_{1, t+1}^{*}\right) w\left(Z^{*, t}, \gamma\right)-\frac{1}{T} \sum_{j=2}^{T-1} g^{\prime}\left(\widehat{u}_{1, j+1}^{(t)}\right) w\left(Z^{j}, \gamma\right)\right)$ has the same limiting distribution of the same functional of $\frac{1}{P^{1 / 2}} \sum_{t=R}^{T-1}\left(g^{\prime}\left(u_{1, t+1}\right) w\left(Z^{t}, \gamma\right)-E\left(g^{\prime}\left(u_{1, t+1}\right) w\left(Z^{t}, \gamma\right)\right)\right)$. Finally, note that $\frac{1}{P^{1 / 2}} \sum_{t=R}^{T-1}\left(\nabla_{\theta} g^{\prime}\left(\bar{u}_{1, t+1}^{*}\right) w\left(Z^{*, t}, \gamma\right)\right)\left(\widetilde{\theta}_{1, t}^{*}-\widehat{\theta}_{1, t}\right)$ properly captures the contribution of recursive parameter estimation error to the covariance kernel. $\square$ 


\section{References}

Altissimo, F. and V. Corradi, (2002), Bounds for Inference with Nuisance Parameters Present only under the Alternative", Econometrics Journal, 5, 494-518.

Andrews, D.W.K., (2002), Higher-Order Improvements of a Computationally Attractive $k$-step Bootstrap for Extremum Estimators, Econometrica, 70, 119-162.

Andrews, D.W.K., (2004), The Block-Block Bootstrap: Improved Asymptotic Refinements, Econometrica, 72, 673-700.

Bachmeier, L. and N.R. Swanson, (2005), Predicting Inflation: Does The Quantity Theory Help?, Economic Inquiry, forthcoming.

Bierens, H.B., (1982): Consistent model specification tests, Journal of Econometrics, 20, 105-134.

Bierens, H.B., (1990): A Conditional Moment Test of Functional Form, Econometrica , 58, 14431458.

Bierens, H.J. and W. Ploberger, (1997): Asymptotic theory of integrated conditional moment tests, Econometrica, 65, 1129-1152.

Carlstein, E. (1986), The Use of Subseries Methods for Estimating the Variance of a General Statistic from a Stationary Time Series, Annals of Statistics, 14, 1171-1179.

Chao, J.C., V. Corradi, and N.R. Swanson (2001), An Out of Sample Test for Granger Causality", Macroeconomic Dynamics, v.5, 598-620.

Christoffersen, P. and F.X. Diebold, (1996), Further Results on Forecasting and Model Selection under Asymmetric Loss, Journal of Applied Econometrics, 11, 561-572.

Christoffersen, P. and F.X. Diebold, (1997), Optimal Prediction Under Asymmetric Loss, Econometric Theory, 13, 808-81\%.

Clark, T.E., and M.W., McCracken, (2001), Tests of Equal Forecast Accuracy and Encompassing for Nested Models, Journal of Econometrics 105, 85-110.

Clark, T.E., and M.W., McCracken (2004), Evaluating Long-Horizon Forecasts, Working Paper, University of Missouri-Columbia.

Corradi, V., (1999), Deciding between $I(0)$ and $I(1)$ via FLIL-Based Bounds, Econometric Theory, 15, 643-663.

Corradi, V. and N.R. Swanson, (2002), A Consistent Test for Out of Sample Nonlinear Predictive Ability, Journal of Econometrics, 110, 353-381.

Corradi, V. and N.R. Swanson, (2004a), "Predictive Density Evaluation", in: Handbook of Economic Forecasting, eds. Clive W.J. Granger, Graham Elliot and Allan Timmerman, Elsevier, Amsterdam, forthcoming.

Corradi, V. and N.R. Swanson, (2004b), Some Recent Developments in Predictive Accuracy Testing with Nested Models and (Generic) Nonlinear Alternatives", International Journal of Forecasting, 20, 185-199.

Corradi, V. and N.R. Swanson, (2005), "Predictive Density and Confidence Intervals Accuracy Tests", Journal of Econometrics, forthcoming.

Diebold, F.X., and R.S. Mariano, (1995), Comparing Predictive Accuracy, Journal of Business and Economic Statistics, 13, 253-263. 
Diebold, F.X. and C. Chen, (1996), Testing Structural Stability with Endogenous Breakpoint: A Size Comparison of Analytic and Bootstrap Procedures, Journal of Econometrics, 70, 221-241.

Elliott, G. and A. Timmerman, (2004a), Optimal Forecast Combinations Under General Loss Functions and Forecast Error Distributions, Journal of Econometrics, forthcoming.

Elliott, G. and A. Timmerman, (2004b), Optimal Forecast Combination Under Regime Switching, International Economic Review, forthcoming.

Fitzenberger, B. (1997), The Moving Block Bootstrap and Robust Inference for Linear Least Square and Quantile Regressions, Journal of Econometrics, 82, 235-287.

Gallant, A.R. and H. White, (1988), A Unified Theory of Estimation and Inference for Nonlinear Dynamic Models, Blackwell, Oxford.

Giacomini, R., and H. White (2003), Conditional Tests for Predictive Ability, manuscript, University of California, San Diego.

Goncalves, S., and H. White, (2004), Maximum Likelihood and the Bootstrap for Nonlinear Dynamic Models, Journal of Econometrics, 119, 199-219.

Granger, C.W.J., (1993), On the Limitations of Comparing Mean Squared Forecast Errors: Comment, Journal of Forecasting, 12, 651-652.

Granger, C.W.J., (1999), Outline of Forecast Theory Using Generalized Cost Functions, Spanish Economic Review, 1, 161-173.

Hall, P., and J.L. Horowitz, (1996), Bootstrap Critical Values for Tests Based on Generalized Method of Moments Estimators, Econometrica, 64, 891-916.

Hansen, B.E., (1996), Inference When a Nuisance Parameter is Not Identified Under the Null Hypothesis, Econometrica, 64, 413-430.

Harvey, D.I., S.J. Leybourne and P. Newbold, (1997), Tests for Forecast Encompassing, Journal of Business and Economic Statistics, 16, 254-259.

Inoue, A., (2001), Testing for Distributional Change in Time Series, Econometric Theory, 17, 156187.

Inoue, A., and M. Shintani, (2004), Bootstrapping GMM Estimators for Time Series, Journal of Econometrics, forthcoming.

Inoue, A., and B. Rossi, (2004), Recursive Predictive Ability Tests for Real Time Data, Working Paper, Duke University and NC State.

Inoue, A. and L. Kilian, (2004), In-Sample and Out-of-Sample Tests of Predictability: Which One Should We Use?, Econometric Reviews, 23, 371-402.

Inoue, A. and L. Kilian, (2005), On the Selection of Forecasting Models, Journal of Econometrics, forthcoming.

Kilian, L., (1999), Exchange Rate and Monetary Fundamentals: What do we Learn from LongHorizon Regressions, Journal of Applied Econometrics, 14, 491-510.

Kilian, L. and M.P. Taylor, (2003), Why is it so Difficult to Beat the Random Walk Forecast of Exchange Rates?, Journal of International Economics, 60, 85-107.

Künsch H.R., (1989), The Jackknife and the Bootstrap for General Stationary Observations, Annals of Statistics, 17, 1217-1241.

Lahiri, S.N., (1999), Theoretical Comparisons of Block Bootstrap Methods, Annals of Statistics, $27,386-404$. 
Linton, O., E. Maasoumi and Y.J. Whang, (2004), Consistent Testing for Stochastic Dominance Under General Sampling Schemes, Review of Economic Studies, forthcoming.

McCracken, M.W., (2004), Asymptotics for Out of Sample Tests of Causality, Working Paper, University of Missouri-Columbia.

McCracken. M.W., and S. Sapp, (2004), Evaluating the Predictive Ability of Exchange Rates Using Long Horizon Regressions: Mind your p's and q's. Journal of Money, Credit and Banking, forthcoming.

Newey, W.K. and K.D. West, (1987), A Simple Positive-Definite Heteroskedasticity and Autocorrelation Consistent Covariance Matrix, Econometrica, 55, 703-708.

Politis, D.N. and J.P. Romano, (1994), The Stationary Bootstrap, Journal of the American Statistical Association, 89, 1303-1313.

Politis, D.N., J.P. Romano and M. Wolf, (1999), Subsampling, Springer and Verlag, New York.

Schorfheide, F., (2004), VAR Forecasting under Misspecification, Journal of Econometrics, forthcoming.

Stinchcombe, M.B. and H. White, (1998), Consistent Specification Testing with Nuisance Parameters Present Only Under the Alternative, Econometric Theory, 14, 3, 295-325.

Swanson, N.R. and H. White, (1997), A Model Selection Approach to Real-Time Macroeconomic Forecasting using Linear Models and Artificial Neural Networks, Review of Economics and Statistics, 79, 540-550.

Weiss, A., (1996) Estimating Time Series Models Using the Relevant Cost Function, Journal of Applied Econometrics, 11, 539-560.

West, K., (1996), Asymptotic Inference About Predictive Ability, Econometrica, 64, 1067-1084.

White, H., (2000), A Reality Check for Data Snooping, Econometrica, 68, 1097-1126.

Wooldridge, J.M. and H. White, (1988), Some Invariance Principles and Central Limit Theorems for Dependent and Heterogeneous Processes, Econometric Theory, 4, 210-230.

Zellner, A., (1986), Bayesian Estimation and Prediction Using Asymmetric Loss Function, Journal of the American Statistical Association, 81, 446-451. 
Table 1: Test Statistics, Sampling Scheme, and Data Generating Processes Used in Empirical and Monte Carlo Sections

\section{Panel A: Test Statistic Mnemonics and Definitions}

$F$ - The standard Wald version of the in-sample F-test is calculated using the entire sample of $T$ observations. In particular, we use: $\left.F=T\left(\sum_{t=1}^{T} \widehat{u}_{1, t}^{2}-\sum_{t=1}^{T} \widehat{u}_{2, t}^{2}\right) / \sum_{t=1}^{T} \widehat{u}_{2, t}^{2}\right)$, where $\widehat{u}_{1, t}$ and $\widehat{u}_{2, t}$ are the in-sample residuals associated with least squares estimation of the small and big models, respectively, and where $T$ denotes the sample size.

$C M$ - The Clark and McCracken (2004) test outlined in Section 5.

$D M$ - The Diebold and Mariano (1995) test outlined in Section 5.

$C S$ - The Corradi and Swanson (2002) test outlined in Section 4.

$C C S$ - The Chao, Corradi and Swanson (2001) test discussed in Section 5.

\section{Panel B: Data Generating Processes Used in Monte Carlo Experiments}

\footnotetext{
$x_{t}=a_{1}+a_{2} x_{t-1}+u_{1, t}, u_{1, t} \sim \operatorname{iidN}(0,1)$

$w_{t}=a_{1}+a_{3} w_{t-1}+u_{2, t}, u_{2, t} \sim \operatorname{iidN}(0,1)$

Size1: $y_{t}=a_{1}+a_{2} y_{t-1}+a_{4} w_{t-1}+u_{3, t}, u_{3, t} \sim i i d N(0,1)$

Size2: $y_{t}=a_{1}+a_{2} y_{t-1}+a_{4} w_{t-1}+a_{3} u_{3, t-1}+u_{3, t}$

Power1: $y_{t}=a_{1}+a_{2} y_{t-1}+2 \exp \left(\tan ^{-1}\left(x_{t-1} / 2\right)\right)+a_{4} w_{t-1}+u_{3, t}$

Power2: $y_{t}=a_{1}+a_{2} y_{t-1}+2 x_{t-1}+a_{4} w_{t-1}+u_{3, t}$

Power3 : $y_{t}=a_{1}+a_{2} y_{t-1}+2 x_{t-1} 1\left\{x_{t-1}>a_{1} /\left(1-a_{2}\right)\right\}+a_{4} w_{t-1}+u_{3, t}$

Power4: $y_{t}=a_{1}+a_{2} y_{t-1}+2 \exp \left(\tan ^{-1}\left(x_{t-1} / 2\right)\right)+a_{4} w_{t-1}+a_{3} u_{3, t-1}+u_{3, t}$

Power5: $y_{t}=a_{1}+a_{2} y_{t-1}+2 x_{t-1}+a_{4} w_{t-1}+a_{3} u_{3, t-1}+u_{3, t}$

Power6: $y_{t}=a_{1}+a_{2} y_{t-1}+2 x_{t-1} 1\left\{x_{t-1}>a_{1} /\left(1-a_{2}\right)\right\}+a_{4} w_{t-1}+a_{3} u_{3, t-1}+u_{3, t}$.

Power7 : $y_{t}=a_{1}+a_{2} y_{t-1}+2 \exp \left(x_{t-1}\right)+a_{4} w_{t-1}+u_{3, t}$

Power8: $y_{t}=a_{1}+a_{2} y_{t-1}+2 x_{t-1}^{2}+a_{4} w_{t-1}+u_{3, t}$

Power9: $y_{t}=a_{1}+a_{2} y_{t-1}+2\left|x_{t-1}\right|+a_{4} w_{t-1}+u_{3, t}$

Power10: $y_{t}=a_{1}+a_{2} y_{t-1}+2 \exp \left(x_{t-1}\right)+a_{4} w_{t-1}+a_{3} u_{3, t-1}+u_{3, t}$

Power11: $y_{t}=a_{1}+a_{2} y_{t-1}+2 x_{t-1}^{2}+a_{4} w_{t-1}+a_{3} u_{3, t-1}+u_{3, t}$

Power12: $y_{t}=a_{1}+a_{2} y_{t-1}+2\left|x_{t-1}\right|+a_{4} w_{t-1}+a_{3} u_{3, t-1}+u_{3, t}$.

Note that the benchmark or "small" model in our test statistic calculations is always $y_{t}=\alpha+\beta y_{t-1}+\epsilon_{t}$; and the "big" model is the same, but with $x_{t-1}$ added as an additional regressor. Note further that $w_{t-1}$ is never used in test statistic forecast model construction. Hence, the difference between the variance of the "small" model and the optimal variance of the "big" model simply the variance of the nonlinear term in the true DGP (whenver the true DGP is either Power1-Power12).
} 
Table 2: Rejection Frequencies of CS Test with $a_{4}=0, T=600, P=0.5 T^{*}$

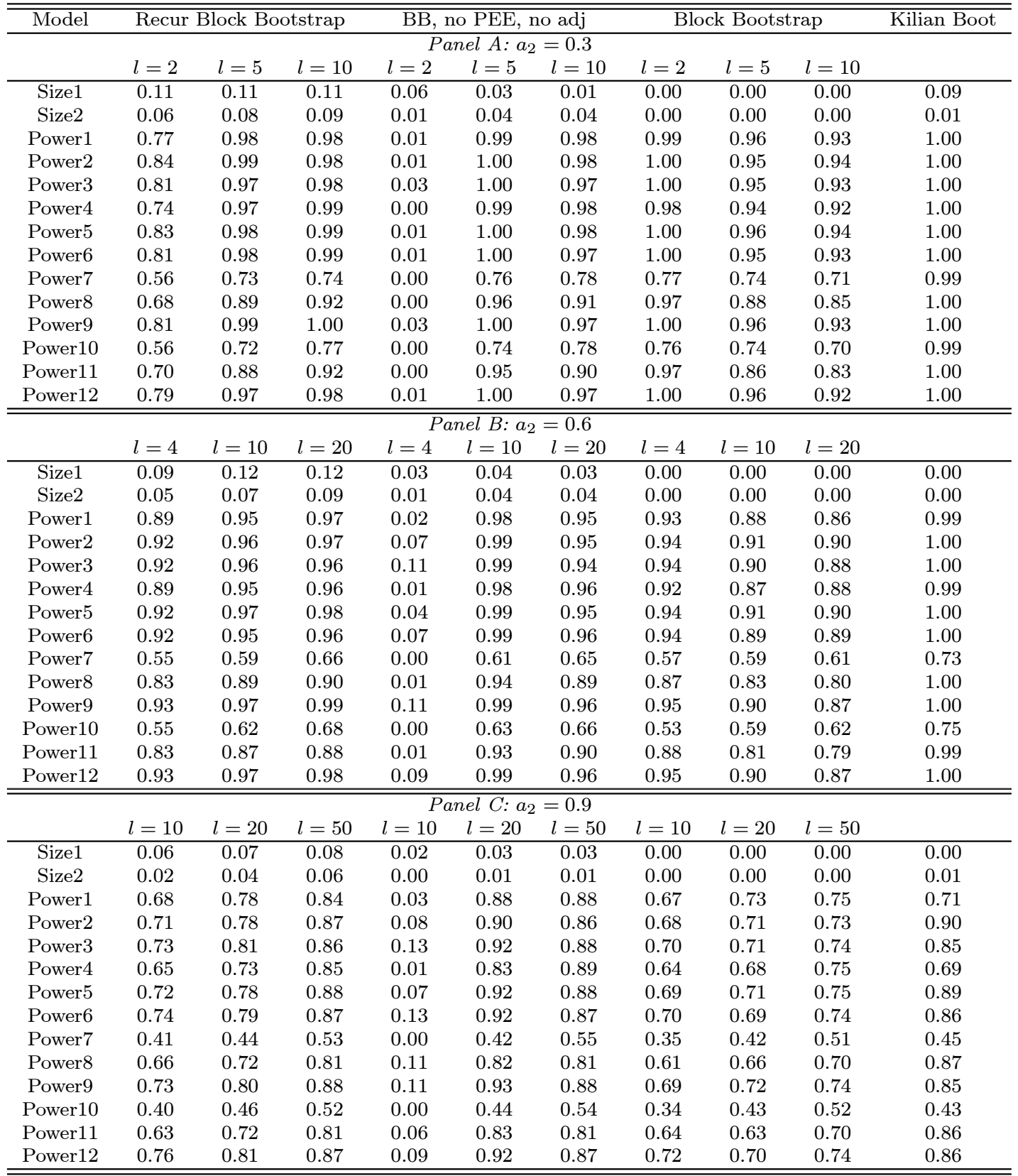

* Notes: All entries are rejection frequencies of the null hypothesis of equal predictive accuracy based on $10 \%$ nominal size critical values constructed using the bootstrap approaches discussed above, where $l$ denotes the block length, and empirical bootstrap distributions are constructed using 100 bootstrap statistics. In particular, "Recur Block Bootstrap" is the bootstrap developed in this paper, "BB, no PEE, no adj" is a naive block bootstrap where no parameter estimation error is assumed, and no recentering (i.e. adjustment) is done in parameter estimation or bootstrap statistic construction, "Block Bootstrap" is the usual block bootstrap that allows for parameter estimation error, but does not recenter parameter estimates or bootstrap statistics, AND "Kilian Boot" is the Kilian (1999) parametric bootstrap implemented in Clark and McCracken (2004) and McCracken and Sapp (2004).. For all models denoted Poweri, $i=1, \ldots, 6$, data are generated with (non) linear Granger causality (see above for further discussion of DGPs. In all experiments, the ex ante forecast period is of length $P$, which is set equal to $(1 / 2) T$, where $T$ is the sample size. All models are estimated recursively, so that parameter estimates are updated before each new prediction is constructed. All reported results are based on 500 Monte Carlo simulations. See Table 1 and Section 5 for further details. 
Table 3: Rejection Frequencies of CS Test with $a_{4}=1, T=600, P=0.5 T^{*}$

\begin{tabular}{|c|c|c|c|c|c|c|c|c|c|c|}
\hline Model & \multicolumn{3}{|c|}{ Recur Block Bootstrap } & \multirow{2}{*}{\multicolumn{3}{|c|}{$\begin{array}{r}\text { BB, no PEE, no adj } \\
\text { Panel } A: a_{2}=0.3\end{array}$}} & \multicolumn{3}{|c|}{ Block Bootstrap } & \multirow[t]{3}{*}{ Kilian Boot } \\
\hline & & & & & & & & & \multirow[b]{2}{*}{$l=10$} & \\
\hline & $l=2$ & $l=5$ & $l=10$ & $l=2$ & $l=5$ & $l=10$ & $l=2$ & $l=5$ & & \\
\hline Size1 & 0.11 & 0.11 & 0.10 & 0.03 & 0.06 & 0.02 & 0.00 & 0.00 & 0.00 & 0.04 \\
\hline Size2 & 0.07 & 0.09 & 0.11 & 0.01 & 0.07 & 0.04 & 0.00 & 0.00 & 0.00 & 0.01 \\
\hline Power1 & 0.61 & 0.91 & 0.93 & 0.00 & 0.96 & 0.95 & 0.90 & 0.90 & 0.86 & 0.98 \\
\hline Power2 & 0.78 & 0.97 & 0.98 & 0.00 & 0.99 & 0.96 & 0.99 & 0.94 & 0.90 & 1.00 \\
\hline Power3 & 0.79 & 0.97 & 0.97 & 0.00 & 0.98 & 0.96 & 0.98 & 0.94 & 0.90 & 1.00 \\
\hline Power4 & 0.59 & 0.91 & 0.95 & 0.00 & 0.95 & 0.95 & 0.88 & 0.91 & 0.86 & 0.98 \\
\hline Power5 & 0.79 & 0.96 & 0.98 & 0.00 & 0.99 & 0.97 & 0.99 & 0.94 & 0.90 & 1.00 \\
\hline Power6 & 0.76 & 0.97 & 0.98 & 0.00 & 0.99 & 0.96 & 0.99 & 0.94 & 0.91 & 1.00 \\
\hline Power7 & 0.60 & 0.75 & 0.77 & 0.00 & 0.74 & 0.79 & 0.76 & 0.74 & 0.72 & 0.95 \\
\hline Power8 & 0.70 & 0.86 & 0.88 & 0.00 & 0.94 & 0.88 & 0.97 & 0.85 & 0.82 & 0.99 \\
\hline Power9 & 0.76 & 0.95 & 0.97 & 0.00 & 0.98 & 0.95 & 0.98 & 0.93 & 0.89 & 1.00 \\
\hline Power10 & 0.61 & 0.75 & 0.78 & 0.00 & 0.74 & 0.78 & 0.78 & 0.73 & 0.72 & 0.96 \\
\hline Power11 & 0.70 & 0.86 & 0.89 & 0.00 & 0.94 & 0.89 & 0.96 & 0.85 & 0.81 & 0.99 \\
\hline Power12 & 0.77 & 0.96 & 0.98 & 0.00 & 0.99 & 0.96 & 0.98 & 0.93 & 0.90 & 0.99 \\
\hline \multicolumn{11}{|c|}{ Panel $B: a_{2}=0.6$} \\
\hline & $l=4$ & $l=10$ & $l=20$ & $l=4$ & $l=10$ & $l=20$ & $l=4$ & $l=10$ & $l=20$ & \\
\hline Size1 & 0.07 & 0.08 & 0.10 & 0.02 & 0.06 & 0.02 & 0.00 & 0.00 & 0.00 & 0.00 \\
\hline Size2 & 0.05 & 0.07 & 0.09 & 0.02 & 0.06 & 0.03 & 0.00 & 0.00 & 0.00 & 0.00 \\
\hline Power1 & 0.78 & 0.87 & 0.91 & 0.00 & 0.93 & 0.91 & 0.83 & 0.79 & 0.80 & 0.92 \\
\hline Power2 & 0.88 & 0.94 & 0.95 & 0.02 & 0.98 & 0.94 & 0.90 & 0.86 & 0.85 & 0.99 \\
\hline Power3 & 0.91 & 0.95 & 0.96 & 0.03 & 0.97 & 0.94 & 0.92 & 0.88 & 0.86 & 0.99 \\
\hline Power4 & 0.74 & 0.87 & 0.91 & 0.00 & 0.92 & 0.91 & 0.83 & 0.81 & 0.81 & 0.90 \\
\hline Power5 & 0.90 & 0.94 & 0.96 & 0.00 & 0.98 & 0.95 & 0.92 & 0.86 & 0.86 & 0.99 \\
\hline Power6 & 0.90 & 0.95 & 0.96 & 0.03 & 0.97 & 0.95 & 0.91 & 0.88 & 0.85 & 1.00 \\
\hline Power7 & 0.55 & 0.62 & 0.67 & 0.00 & 0.61 & 0.67 & 0.55 & 0.58 & 0.63 & 0.75 \\
\hline Power8 & 0.78 & 0.85 & 0.86 & 0.01 & 0.93 & 0.85 & 0.84 & 0.75 & 0.72 & 0.97 \\
\hline Power9 & 0.90 & 0.96 & 0.96 & 0.05 & 0.97 & 0.95 & 0.93 & 0.84 & 0.85 & 0.98 \\
\hline Power10 & 0.54 & 0.61 & 0.66 & 0.00 & 0.62 & 0.67 & 0.53 & 0.58 & 0.62 & 0.75 \\
\hline Power11 & 0.78 & 0.83 & 0.88 & 0.01 & 0.91 & 0.86 & 0.83 & 0.75 & 0.74 & 0.97 \\
\hline Power12 & 0.89 & 0.95 & 0.96 & 0.03 & 0.97 & 0.96 & 0.92 & 0.86 & 0.83 & 0.98 \\
\hline \multicolumn{11}{|c|}{ Panel $C: a_{2}=0.9$} \\
\hline & $l=10$ & $l=20$ & $l=50$ & $l=10$ & $l=20$ & $l=50$ & $l=10$ & $l=20$ & $l=50$ & \\
\hline Size1 & 0.02 & 0.05 & 0.05 & 0.00 & 0.03 & 0.02 & 0.00 & 0.00 & 0.00 & 0.00 \\
\hline Size2 & 0.02 & 0.02 & 0.03 & 0.00 & 0.02 & 0.02 & 0.00 & 0.00 & 0.00 & 0.00 \\
\hline Power1 & 0.53 & 0.63 & 0.76 & 0.00 & 0.63 & 0.84 & 0.50 & 0.60 & 0.69 & 0.55 \\
\hline Power2 & 0.69 & 0.76 & 0.84 & 0.02 & 0.88 & 0.85 & 0.68 & 0.71 & 0.72 & 0.82 \\
\hline Power3 & 0.72 & 0.80 & 0.86 & 0.05 & 0.91 & 0.86 & 0.72 & 0.74 & 0.75 & 0.81 \\
\hline Power4 & 0.49 & 0.61 & 0.75 & 0.00 & 0.52 & 0.83 & 0.43 & 0.60 & 0.68 & 0.56 \\
\hline Power5 & 0.69 & 0.76 & 0.82 & 0.01 & 0.88 & 0.85 & 0.66 & 0.69 & 0.74 & 0.82 \\
\hline Power6 & 0.73 & 0.76 & 0.84 & 0.07 & 0.89 & 0.86 & 0.71 & 0.70 & 0.74 & 0.81 \\
\hline Power7 & 0.40 & 0.44 & 0.50 & 0.01 & 0.39 & 0.52 & 0.34 & 0.43 & 0.49 & 0.41 \\
\hline Power8 & 0.61 & 0.66 & 0.77 & 0.08 & 0.81 & 0.77 & 0.61 & 0.63 & 0.66 & 0.85 \\
\hline Power9 & 0.70 & 0.75 & 0.82 & 0.10 & 0.88 & 0.84 & 0.66 & 0.67 & 0.70 & 0.85 \\
\hline Power10 & 0.41 & 0.45 & 0.53 & 0.01 & 0.42 & 0.56 & 0.33 & 0.43 & 0.51 & 0.40 \\
\hline Power11 & 0.64 & 0.69 & 0.76 & 0.07 & 0.79 & 0.75 & 0.64 & 0.62 & 0.66 & 0.85 \\
\hline Power12 & 0.68 & 0.77 & 0.85 & 0.08 & 0.88 & 0.85 & 0.66 & 0.68 & 0.71 & 0.82 \\
\hline
\end{tabular}

* Notes: See notes to Table 2. 
Table 4: Rejection Frequencies of Various Tests with $a_{4}=0, T=600, P=0.5 T^{*}$

\begin{tabular}{|c|c|c|c|c|c|c|c|c|c|c|c|c|}
\hline \multirow[t]{2}{*}{$\overline{\bar{M} \text { Model }}$} & \multicolumn{3}{|c|}{ Assume $\pi=0$} & \multicolumn{2}{|c|}{ Assume $\pi>0$} & \multicolumn{4}{|c|}{ Kilian Bootstrap } & \multicolumn{3}{|c|}{ Recur Block Bootstrap } \\
\hline & $\mathrm{F}$ & $\mathrm{DM}$ & $\mathrm{CM}$ & $\mathrm{DM}$ & $\mathrm{CM}$ & $\mathrm{F}$ & $\mathrm{DM}$ & $\mathrm{CM}$ & $\mathrm{CCS}$ & CCS-l1 & $\mathrm{CCS}-\mathrm{l} 2$ & CCS-l3 \\
\hline \multicolumn{13}{|c|}{ Panel $A: a_{2}=0.3$} \\
\hline Size1 & 0.24 & 0.01 & 0.05 & 0.09 & 0.09 & 0.08 & 0.10 & 0.10 & 0.08 & 0.22 & 0.22 & 0.20 \\
\hline Size2 & 0.25 & 0.01 & 0.06 & 0.09 & 0.09 & 0.09 & 0.09 & 0.09 & 0.03 & 0.17 & 0.17 & 0.17 \\
\hline Power1 & 1.00 & 1.00 & 1.00 & 1.00 & 1.00 & 1.00 & 1.00 & 1.00 & 1.00 & 1.00 & 1.00 & 0.99 \\
\hline Power2 & 1.00 & 1.00 & 1.00 & 1.00 & 1.00 & 1.00 & 1.00 & 1.00 & 1.00 & 1.00 & 1.00 & 1.00 \\
\hline Power3 & 1.00 & 1.00 & 1.00 & 1.00 & 1.00 & 1.00 & 1.00 & 1.00 & 1.00 & 1.00 & 1.00 & 0.98 \\
\hline Power4 & 1.00 & 1.00 & 1.00 & 1.00 & 1.00 & 1.00 & 1.00 & 1.00 & 1.00 & 1.00 & 1.00 & 0.99 \\
\hline Power5 & 1.00 & 1.00 & 1.00 & 1.00 & 1.00 & 1.00 & 1.00 & 1.00 & 1.00 & 1.00 & 1.00 & 1.00 \\
\hline Power6 & 1.00 & 1.00 & 1.00 & 1.00 & 1.00 & 1.00 & 1.00 & 1.00 & 1.00 & 1.00 & 1.00 & 0.98 \\
\hline Power7 & 1.00 & 1.00 & 1.00 & 1.00 & 1.00 & 1.00 & 1.00 & 1.00 & 1.00 & 1.00 & 0.87 & 0.78 \\
\hline Power8 & 1.00 & 1.00 & 1.00 & 1.00 & 1.00 & 1.00 & 1.00 & 1.00 & 1.00 & 1.00 & 1.00 & 0.95 \\
\hline Power9 & 1.00 & 1.00 & 1.00 & 1.00 & 1.00 & 1.00 & 1.00 & 1.00 & 1.00 & 1.00 & 1.00 & 1.00 \\
\hline Power10 & 1.00 & 1.00 & 1.00 & 1.00 & 1.00 & 1.00 & 1.00 & 1.00 & 1.00 & 0.99 & 0.87 & 0.78 \\
\hline Power11 & 1.00 & 1.00 & 1.00 & 1.00 & 1.00 & 1.00 & 1.00 & 1.00 & 1.00 & 1.00 & 0.99 & 0.94 \\
\hline Power12 & 1.00 & 1.00 & 1.00 & 1.00 & 1.00 & 1.00 & 1.00 & 1.00 & 1.00 & 1.00 & 1.00 & 1.00 \\
\hline \multicolumn{13}{|c|}{$\overline{\bar{P} \text { Panel } B: a_{2}=0.6}$} \\
\hline Size1 & 0.25 & 0.01 & 0.05 & 0.09 & 0.10 & 0.10 & 0.09 & 0.09 & 0.00 & 0.18 & 0.18 & 0.20 \\
\hline Size2 & 0.27 & 0.02 & 0.06 & 0.11 & 0.10 & 0.10 & 0.10 & 0.10 & 0.00 & 0.12 & 0.15 & 0.17 \\
\hline Power1 & 1.00 & 1.00 & 1.00 & 1.00 & 1.00 & 1.00 & 1.00 & 1.00 & 1.00 & 1.00 & 0.97 & 0.96 \\
\hline Power2 & 1.00 & 1.00 & 1.00 & 1.00 & 1.00 & 1.00 & 1.00 & 1.00 & 1.00 & 1.00 & 0.99 & 0.98 \\
\hline Power3 & 1.00 & 1.00 & 1.00 & 1.00 & 1.00 & 1.00 & 1.00 & 1.00 & 1.00 & 1.00 & 0.97 & 0.94 \\
\hline Power4 & 1.00 & 1.00 & 1.00 & 1.00 & 1.00 & 1.00 & 1.00 & 1.00 & 1.00 & 1.00 & 0.97 & 0.95 \\
\hline Power5 & 1.00 & 1.00 & 1.00 & 1.00 & 1.00 & 1.00 & 1.00 & 1.00 & 1.00 & 1.00 & 0.98 & 0.98 \\
\hline Power6 & 1.00 & 1.00 & 1.00 & 1.00 & 1.00 & 1.00 & 1.00 & 1.00 & 1.00 & 1.00 & 0.97 & 0.96 \\
\hline Power7 & 1.00 & 1.00 & 1.00 & 1.00 & 1.00 & 1.00 & 0.96 & 0.98 & 1.00 & 0.84 & 0.68 & 0.64 \\
\hline Power8 & 1.00 & 1.00 & 1.00 & 1.00 & 1.00 & 1.00 & 1.00 & 1.00 & 1.00 & 1.00 & 0.92 & 0.88 \\
\hline Power9 & 1.00 & 1.00 & 1.00 & 1.00 & 1.00 & 1.00 & 1.00 & 1.00 & 1.00 & 1.00 & 0.98 & 0.96 \\
\hline Power10 & 1.00 & 1.00 & 1.00 & 1.00 & 1.00 & 1.00 & 0.97 & 0.98 & 1.00 & 0.83 & 0.68 & 0.64 \\
\hline Power11 & 1.00 & 1.00 & 1.00 & 1.00 & 1.00 & 1.00 & 1.00 & 1.00 & 1.00 & 1.00 & 0.93 & 0.88 \\
\hline Power12 & 1.00 & 1.00 & 1.00 & 1.00 & 1.00 & 1.00 & 1.00 & 1.00 & 1.00 & 1.00 & 0.98 & 0.96 \\
\hline \multicolumn{13}{|c|}{$\overline{P a n e l ~ C: a_{2}=0.9}$} \\
\hline Size1 & 0.25 & 0.01 & 0.06 & 0.09 & 0.10 & 0.10 & 0.07 & 0.07 & 0.00 & 0.14 & 0.17 & 0.20 \\
\hline Size2 & 0.29 & 0.03 & 0.08 & 0.11 & 0.12 & 0.14 & 0.11 & 0.11 & 0.00 & 0.11 & 0.12 & 0.16 \\
\hline Power1 & 1.00 & 1.00 & 1.00 & 1.00 & 1.00 & 1.00 & 1.00 & 1.00 & 0.81 & 0.85 & 0.86 & 0.88 \\
\hline Power2 & 1.00 & 1.00 & 1.00 & 1.00 & 1.00 & 1.00 & 1.00 & 1.00 & 0.93 & 0.90 & 0.90 & 0.92 \\
\hline Power3 & 1.00 & 1.00 & 1.00 & 1.00 & 1.00 & 1.00 & 1.00 & 1.00 & 0.90 & 0.84 & 0.86 & 0.88 \\
\hline Power4 & 1.00 & 1.00 & 1.00 & 1.00 & 1.00 & 1.00 & 1.00 & 1.00 & 0.82 & 0.85 & 0.86 & 0.89 \\
\hline Power5 & 1.00 & 1.00 & 1.00 & 1.00 & 1.00 & 1.00 & 1.00 & 1.00 & 0.93 & 0.89 & 0.89 & 0.93 \\
\hline Power6 & 1.00 & 1.00 & 1.00 & 1.00 & 1.00 & 1.00 & 1.00 & 1.00 & 0.91 & 0.82 & 0.85 & 0.88 \\
\hline Power7 & 1.00 & 0.98 & 1.00 & 1.00 & 1.00 & 1.00 & 0.85 & 0.91 & 0.73 & 0.45 & 0.47 & 0.47 \\
\hline Power8 & 1.00 & 1.00 & 1.00 & 1.00 & 1.00 & 1.00 & 1.00 & 1.00 & 0.93 & 0.79 & 0.78 & 0.80 \\
\hline Power9 & 1.00 & 1.00 & 1.00 & 1.00 & 1.00 & 1.00 & 1.00 & 1.00 & 0.91 & 0.85 & 0.87 & 0.89 \\
\hline Power10 & 1.00 & 0.98 & 1.00 & 1.00 & 1.00 & 1.00 & 0.84 & 0.92 & 0.74 & 0.47 & 0.45 & 0.47 \\
\hline Power11 & 1.00 & 1.00 & 1.00 & 1.00 & 1.00 & 1.00 & 1.00 & 1.00 & 0.93 & 0.77 & 0.76 & 0.82 \\
\hline Power12 & 1.00 & 1.00 & 1.00 & 1.00 & 1.00 & 1.00 & 1.00 & 1.00 & 0.91 & 0.84 & 0.86 & 0.88 \\
\hline
\end{tabular}

* Notes: See notes to Table 2. Test statistics, denoted by F, DM, CM, CS, and CCS are summarized in Table 1. Block lengths are denoted by $l 1, l 2$, and $l 3$, so that $C C S-l 3$ is the CCS test with block length $l 3$. Block lengths corrspond to those used in Table 2 and 3 , so that for $a_{2}=0.3, l 1, l 2, l 3=2,5,10$. The block lengths for $a_{2}=0.6$ and $a_{2}=0.9$ are $l 1, l 2, l 3=4,10,20$ and $l 1, l 2, l 3=10,20,50$, respectively. $\pi=0$ corresponds to the case where standard critical values based upon the assumption that parameter estimation error vanishes asymptotically are used (i.e. $\pi=\lim _{T->\infty} P / R=0$ ). $\pi>0$ corresponds to the case where nonstandard critical values (see McCracken (2004)) based upon the assumption that parameter estimation error does not vanish asymptotically are used (i.e. $\pi=\lim _{T->\infty} P / R>0$ ). In this case, we assume that $\pi=1$. 
Table 5: Rejection Frequencies of Various Tests with $a_{4}=1, T=600, P=0.5 T^{*}$

\begin{tabular}{|c|c|c|c|c|c|c|c|c|c|c|c|c|}
\hline \multirow[t]{2}{*}{$\overline{\bar{M} \text { Model }}$} & \multicolumn{3}{|c|}{ Assume $\pi=0$} & \multicolumn{2}{|c|}{ Assume $\pi>0$} & \multicolumn{4}{|c|}{ Kilian Bootstrap } & \multicolumn{3}{|c|}{ Recur Block Bootstrap } \\
\hline & $\mathrm{F}$ & $\mathrm{DM}$ & CM & DM & CM & $\mathrm{F}$ & $\mathrm{DM}$ & CM & CCS & CCS- $l 1$ & CCS- $l 2$ & CCS-l3 \\
\hline \multicolumn{13}{|c|}{$\overline{P \text { Panel } A: a_{2}=0.3}$} \\
\hline Size1 & 0.26 & 0.02 & 0.05 & 0.07 & 0.07 & 0.12 & 0.10 & 0.09 & 0.04 & 0.23 & 0.21 & 0.22 \\
\hline Size2 & 0.27 & 0.02 & 0.05 & 0.09 & 0.07 & 0.11 & 0.09 & 0.09 & 0.02 & 0.16 & 0.19 & 0.22 \\
\hline Power1 & 1.00 & 1.00 & 1.00 & 1.00 & 1.00 & 1.00 & 1.00 & 1.00 & 1.00 & 1.00 & 0.99 & 0.97 \\
\hline Power2 & 1.00 & 1.00 & 1.00 & 1.00 & 1.00 & 1.00 & 1.00 & 1.00 & 1.00 & 1.00 & 1.00 & 1.00 \\
\hline Power3 & 1.00 & 1.00 & 1.00 & 1.00 & 1.00 & 1.00 & 1.00 & 1.00 & 1.00 & 1.00 & 1.00 & 0.99 \\
\hline Power4 & 1.00 & 1.00 & 1.00 & 1.00 & 1.00 & 1.00 & 1.00 & 1.00 & 1.00 & 1.00 & 0.99 & 0.97 \\
\hline Power5 & 1.00 & 1.00 & 1.00 & 1.00 & 1.00 & 1.00 & 1.00 & 1.00 & 1.00 & 1.00 & 1.00 & 1.00 \\
\hline Power6 & 1.00 & 1.00 & 1.00 & 1.00 & 1.00 & 1.00 & 1.00 & 1.00 & 1.00 & 1.00 & 1.00 & 0.98 \\
\hline Power7 & 1.00 & 1.00 & 1.00 & 1.00 & 1.00 & 1.00 & 1.00 & 1.00 & 1.00 & 1.00 & 0.88 & 0.76 \\
\hline Power8 & 1.00 & 1.00 & 1.00 & 1.00 & 1.00 & 1.00 & 1.00 & 1.00 & 1.00 & 1.00 & 0.98 & 0.93 \\
\hline Power9 & 1.00 & 1.00 & 1.00 & 1.00 & 1.00 & 1.00 & 1.00 & 1.00 & 1.00 & 1.00 & 1.00 & 0.98 \\
\hline Power10 & 1.00 & 1.00 & 1.00 & 1.00 & 1.00 & 1.00 & 1.00 & 1.00 & 1.00 & 1.00 & 0.88 & 0.76 \\
\hline Power11 & 1.00 & 1.00 & 1.00 & 1.00 & 1.00 & 1.00 & 1.00 & 1.00 & 1.00 & 1.00 & 0.98 & 0.93 \\
\hline Power12 & 1.00 & 1.00 & 1.00 & 1.00 & 1.00 & 1.00 & 1.00 & 1.00 & 1.00 & 1.00 & 1.00 & 0.98 \\
\hline \multicolumn{13}{|c|}{ Panel B: $a_{2}=0.6$} \\
\hline Size1 & 0.26 & 0.02 & 0.05 & 0.11 & 0.08 & 0.10 & 0.09 & 0.09 & 0.01 & 0.15 & 0.17 & 0.20 \\
\hline Size2 & 0.27 & 0.02 & 0.06 & 0.09 & 0.08 & 0.11 & 0.10 & 0.10 & 0.00 & 0.10 & 0.16 & 0.18 \\
\hline Power1 & 1.00 & 1.00 & 1.00 & 1.00 & 1.00 & 1.00 & 1.00 & 1.00 & 1.00 & 0.99 & 0.94 & 0.89 \\
\hline Power2 & 1.00 & 1.00 & 1.00 & 1.00 & 1.00 & 1.00 & 1.00 & 1.00 & 1.00 & 1.00 & 0.99 & 0.97 \\
\hline Power3 & 1.00 & 1.00 & 1.00 & 1.00 & 1.00 & 1.00 & 1.00 & 1.00 & 1.00 & 0.99 & 0.96 & 0.94 \\
\hline Power4 & 1.00 & 1.00 & 1.00 & 1.00 & 1.00 & 1.00 & 1.00 & 1.00 & 1.00 & 0.98 & 0.93 & 0.91 \\
\hline Power5 & 1.00 & 1.00 & 1.00 & 1.00 & 1.00 & 1.00 & 1.00 & 1.00 & 1.00 & 1.00 & 0.99 & 0.96 \\
\hline Power6 & 1.00 & 1.00 & 1.00 & 1.00 & 1.00 & 1.00 & 1.00 & 1.00 & 1.00 & 1.00 & 0.97 & 0.95 \\
\hline Power7 & 1.00 & 1.00 & 1.00 & 1.00 & 1.00 & 1.00 & 0.97 & 0.99 & 0.99 & 0.82 & 0.68 & 0.63 \\
\hline Power8 & 1.00 & 1.00 & 1.00 & 1.00 & 1.00 & 1.00 & 1.00 & 1.00 & 1.00 & 0.98 & 0.90 & 0.87 \\
\hline Power9 & 1.00 & 1.00 & 1.00 & 1.00 & 1.00 & 1.00 & 1.00 & 1.00 & 1.00 & 0.99 & 0.97 & 0.94 \\
\hline Power10 & 1.00 & 1.00 & 1.00 & 1.00 & 1.00 & 1.00 & 0.96 & 0.99 & 0.99 & 0.80 & 0.66 & 0.63 \\
\hline Power11 & 1.00 & 1.00 & 1.00 & 1.00 & 1.00 & 1.00 & 1.00 & 1.00 & 1.00 & 0.98 & 0.91 & 0.87 \\
\hline Power12 & 1.00 & 1.00 & 1.00 & 1.00 & 1.00 & 1.00 & 1.00 & 1.00 & 1.00 & 0.99 & 0.96 & 0.95 \\
\hline \multicolumn{13}{|c|}{ Panel C: $a_{2}=0.9$} \\
\hline Size1 & 0.27 & 0.03 & 0.08 & 0.12 & 0.12 & 0.13 & 0.09 & 0.11 & 0.00 & 0.09 & 0.12 & 0.15 \\
\hline Size2 & 0.27 & 0.03 & 0.09 & 0.11 & 0.12 & 0.14 & 0.11 & 0.13 & 0.00 & 0.07 & 0.11 & 0.16 \\
\hline Power1 & 1.00 & 1.00 & 1.00 & 1.00 & 1.00 & 1.00 & 1.00 & 1.00 & 0.71 & 0.76 & 0.77 & 0.82 \\
\hline Power2 & 1.00 & 1.00 & 1.00 & 1.00 & 1.00 & 1.00 & 1.00 & 1.00 & 0.89 & 0.86 & 0.88 & 0.89 \\
\hline Power3 & 1.00 & 1.00 & 1.00 & 1.00 & 1.00 & 1.00 & 1.00 & 1.00 & 0.88 & 0.85 & 0.84 & 0.86 \\
\hline Power4 & 1.00 & 1.00 & 1.00 & 1.00 & 1.00 & 1.00 & 1.00 & 1.00 & 0.72 & 0.71 & 0.77 & 0.81 \\
\hline Power5 & 1.00 & 1.00 & 1.00 & 1.00 & 1.00 & 1.00 & 1.00 & 1.00 & 0.90 & 0.86 & 0.85 & 0.89 \\
\hline Power6 & 1.00 & 1.00 & 1.00 & 1.00 & 1.00 & 1.00 & 1.00 & 1.00 & 0.88 & 0.81 & 0.82 & 0.85 \\
\hline Power7 & 1.00 & 0.96 & 1.00 & 0.98 & 1.00 & 1.00 & 0.85 & 0.93 & 0.74 & 0.46 & 0.43 & 0.46 \\
\hline Power8 & 1.00 & 1.00 & 1.00 & 1.00 & 1.00 & 1.00 & 1.00 & 1.00 & 0.93 & 0.73 & 0.74 & 0.76 \\
\hline Power9 & 1.00 & 1.00 & 1.00 & 1.00 & 1.00 & 1.00 & 1.00 & 1.00 & 0.89 & 0.79 & 0.82 & 0.84 \\
\hline Power10 & 1.00 & 0.96 & 1.00 & 0.98 & 1.00 & 1.00 & 0.84 & 0.93 & 0.74 & 0.46 & 0.44 & 0.48 \\
\hline Power11 & 1.00 & 1.00 & 1.00 & 1.00 & 1.00 & 1.00 & 1.00 & 1.00 & 0.93 & 0.74 & 0.75 & 0.75 \\
\hline Power12 & 1.00 & 1.00 & 1.00 & 1.00 & 1.00 & 1.00 & 1.00 & 1.00 & 0.88 & 0.80 & 0.81 & 0.85 \\
\hline
\end{tabular}

* Notes: See notes to Table 4. 


\section{Table 6: Mean Square Forecast Errors Based Indicating the Marginal Predictive}

Content of Unemployment for the CPI*

\begin{tabular}{cccc}
\hline \hline Start Year & & Case 1 & Case 2 \\
\hline \hline & small model & big model & big model \\
\hline 1965 & 0.6239 & 0.6316 & 0.6289 \\
1966 & 0.6329 & 0.6413 & 0.6368 \\
1967 & 0.6335 & 0.6449 & 0.6370 \\
1968 & 0.6363 & 0.6511 & 0.6403 \\
1969 & 0.6363 & 0.6591 & 0.6399 \\
1970 & 0.6267 & 0.6599 & 0.6305 \\
1971 & 0.6295 & 0.6596 & 0.6363 \\
1972 & 0.6438 & 0.6703 & 0.6507 \\
1973 & 0.6564 & 0.6816 & 0.6636 \\
1974 & 0.5603 & 0.5854 & 0.5650 \\
1975 & 0.4929 & 0.5038 & 0.4993 \\
1976 & 0.4849 & 0.4864 & 0.4916 \\
1977 & 0.4953 & 0.4963 & 0.5026 \\
1978 & 0.4933 & 0.4944 & 0.4979 \\
1979 & 0.4882 & 0.4900 & 0.4905 \\
1980 & 0.4598 & 0.4619 & 0.4610 \\
1981 & 0.4226 & 0.4267 & 0.4246 \\
1982 & 0.4158 & 0.4213 & 0.4178 \\
1983 & 0.3810 & 0.3813 & 0.3820 \\
1984 & 0.3799 & 0.3799 & 0.3815 \\
1985 & 0.3873 & 0.3870 & 0.3887 \\
1986 & 0.3982 & 0.3977 & 0.3995 \\
1987 & 0.3751 & 0.3740 & 0.3755 \\
1988 & 0.3881 & 0.3870 & 0.3887 \\
1989 & 0.4008 & 0.3996 & 0.4018 \\
1990 & 0.4102 & 0.4089 & 0.4110 \\
1991 & 0.3796 & 0.3781 & 0.3814 \\
1992 & 0.3915 & 0.3897 & 0.3930 \\
1993 & 0.4117 & 0.4093 & 0.4131 \\
1994 & 0.4329 & 0.4301 & 0.4346 \\
1995 & 0.4559 & 0.4528 & 0.4586 \\
\hline \hline
\end{tabular}

* Notes: Mean square forecast errors (multiplied by 100000) are reported for two models (small which contains only lags of inflation, and big which contains lags of inflation and unemployment). Case 1 refers to a big model that is specified using inflation and unemployment, and Case 2 refers to the same, but specified using inflation and differenced unemployment. All predictions are of 1-step ahead inflation, and predictive periods begin in the year given in the first column of entries in the table. All estmation and prediction is done recursively, as detailed in Section 6. 


\section{Table 7: Tests for the Marginal Predictive Content of Unemployment for the CPI*}

\begin{tabular}{|c|c|c|c|c|}
\hline \multirow[t]{2}{*}{ Test Statistic } & \multicolumn{2}{|c|}{ Prediction Period Begins 1970:1 } & \multicolumn{2}{|c|}{ Prediction Period Begins 1984:1 } \\
\hline & Unem & $\Delta U n e m$ & Unem & $\Delta U n e m$ \\
\hline CS (Recur Block Bootstrap) & reject & reject & no reject & no reject \\
\hline CS (Kilian Bootstrap) & reject & reject & no reject & no reject \\
\hline CCS (Recur Block Bootstrap) & reject & reject & reject & no reject \\
\hline CCS (Kilian Bootstrap) & no reject & no reject & no reject & no reject \\
\hline $\mathrm{F}$ & no reject & no reject & no reject & no reject \\
\hline DM (Kilian Bootstrap) & no reject & no reject & no reject & no reject \\
\hline CM (Kilian Bootstrap) & no reject & no reject & no reject & no reject \\
\hline
\end{tabular}

* Notes: Entries denote either rejection (reject), or failure to reject (no reject) the null hypothesis that unemployment (columns $2,4)$ or differenced unemployment (columns 3,5) has no marginal predictive content for core U.S. CPI. Starred entries denote nominal $10 \%$ level test rejection based on the use of critical values constructed using the approach signified using the mnemonic in brackets in the first column of the table. Explanation of these mnemonics are given in the notes to Table 2 and 4 . All models use monthly data and all predictions are 1-step ahead and are constructed using recursively estimated models. Results are given for predictions periods beginning with either 1970:1 or 1984:1 and ending in 2004:12. Estimation periods always begin in 1955:1. See Section 6 for complete details. has 